\title{
Diversity of Melon Accessions from Northeastern Brazil and Their Relationships with Germplasms of Diverse Origins
}

\author{
Ana Carolina de Assis Dantas and Ioná Santos Araújo Holanda \\ Department of Crop Science, Federal Rural University of Semi-Arid, P.O. Box 137, Mossoró, Rio \\ Grande do Norte 59631-180, Brazil \\ Cristina Esteras \\ Institute for the Conservation and Breeding of Agricultural Biodiversity (COMAV), Universitat \\ Politècnica de Valencia, Camino de Vera, 8E, Escalera J, Valencia 46022, Spain
}

Glauber Henrique de Sousa Nunes

Department of Crop Science, Federal Rural University of Semi-Arid, P.O. Box 137, Mossoró, Rio

Grande do Norte 59631-180, Brazil

\author{
Maria Belén Picó ${ }^{1}$ \\ Institute for the Conservation and Breeding of Agricultural Biodiversity (COMAV), Universitat \\ Politècnica de Valencia, Camino de Vera, 8E, Escalera J, Valencia 46022, Spain
}

\begin{abstract}
AdDitional INDEX words. Cucumis melo, microsatellite, phenotyping, genetic diversity, breeding
Abstract. Melon (Cucumis melo) is one of the most important horticultural crops in Brazil. A set of 40 melon landraces representing a northeastern Brazilian variation was agronomically evaluated with 13 morphological traits and molecularly with 13 simple sequence repeat markers and compared with a set of 21 reference accessions of diverse taxonomic and geographic origins. Brazilian accessions were highly variable for seed, flowering time, fruit, and yieldrelated traits. However, most of them shared a monoecious sex type, a climacteric ripening behavior, and fruit that were low to medium in sugar with a high acid content, characteristics in accordance with those of Asian melons belonging to Momordica, Flexuosus, and Chate, and Far-Eastern Makuwa and Chinensis melon groups. Molecular analysis confirmed the genetic similarity of the Brazilian landraces to reference accessions from India, the Far East, and the Middle East belonging to those botanical groups. Momordica, Makuwa, and Chinensis melons are rarely found as landraces outside of this area thus suggesting that these landraces were introduced into Brazil from these regions. Our molecular results also support a high genetic diversity in the Brazilian collection [polymorphism information content $(P I C)=0.43$ ], which is only slightly lower than that of the references $(P I C=0.59)$ that include most of the main horticultural groups of the two $C$. melo subspecies. This study shows that in Brazil, there is currently a large variability of this species maintained in the form of landraces, which are a potentially useful resource for breeding melons.
\end{abstract}

Melon is a morphologically diverse diploid species $(2 n=2 x=$ $24)$ and a crop of great commercial importance with many varied uses, depending on the type of fruit. It is mainly cultivated in tropical and subtropical regions, but its cultivation has been increasingly extended to temperate climate regions. In 2012, the world melon fruit production was near 30 million tons [Food and Agriculture Organization of the United Nations (FAO), 2014].

The geographic origin of melon is still controversial. Despite the great genetic diversity of Indian and east-central Asian landraces, modern authors have maintained that this species is of African origin on the basis that it shares the same number of chromosomes with many African wild Cucumis species (Dhillon et al., 2007; Kerje and Grum, 2000; Luan et al., 2008; Robinson and Decker-Walters, 1997). However, recent systematic studies (John et al., 2012; Sebastian et al., 2010 ), covering the entire natural range of the genus

Received for publication 13 Jan. 2015. Accepted for publication 30 July 2015. The authors thank CAPES-MINECO projects 294/13, PHB2012-0098, and PHBP14/00021.

${ }^{1}$ Corresponding author. E-mail: mpicosi@btc.upv.es.
Cucumis, indicate that the Australian Cucumis picrocarpus is sister to C. melo, of which the wild progenitors are likely Cucumis trigonus and Cucumis callosus, which are both Asian.

Melon may have been domesticated primarily due to the nutritional value of its seeds, and it later went through an intense process of diversification (Pitrat, 2008). As a result of this process, melon became the most polymorphic species in the genus (Luan et al., 2010), with a large diversity in vine and fruit traits (Esteras et al., 2012; Stepansky et al., 1999). Melon diversity is spread in primary and secondary centers. The primary centers are located in central and southern Asia (Tzitzikas et al., 2009). Far-Eastern Asian and Mediterranean regions comprise the secondary centers (Blanca et al., 2012; López-Sesé et al., 2003; Monforte et al., 2003).

Cucumis melo is divided into two subspecies, melo and agrestis (Jeffrey, 1980; Kirkbride 1993). Attempts to group melon cultivars date back to the classic study by Naudin (1859), in which the species was subdivided into nine tribes of cultivated melons and one wild form. This pioneering work became the basis for subsequent classifications (Burger et al., 2010; Pitrat, 2008; Pitrat et al., 2000). These authors reviewed 
the previous literature and proposed similar melon groups but differed in their botanical classification below the subspecies level, whereas Pitrat et al. (2000) and Pitrat (2008) used the term botanical group or botanical variety for each of the members of the two subspecies, Burger et al. (2010) classified them into horticultural groups, 11 in ssp. melo, including Inodorus, Cantalupensis, Reticulatus, Adana, Ameri, Chandalak, Chate, Flexuosus, Dudaim, Chito, and Tibish and 5 in ssp. agrestis, including Conomon, Makuwa, Chinensis, Acidulus, and Momordica. However, because all of them are fully crossable, many intermediate types exist, often making classification difficult.

In general, molecular analyses support sub-specific classification (Blanca et al., 2012; Esteras et al., 2013; Leida et al., 2015; Monforte et al., 2003), although Chito and Tibish are assigned to ssp. agrestis, and the Asian melons, Dudaim, Momordica, and the elongated Flexuosus and Chate melons, used in ancient times, are placed in an intermediate position between both subspecies. A high molecular diversity and a high degree of genetic admixture is found in the Adana, Chandalack, and Ameri groups, which represent old Western and Central Asian landraces from which the main ssp. melo groups, Inodorus, Cantalupensis, and Reticulatus, may have been derived (Leida et al., 2015; Nesom, 2011). These ssp. melo groups form molecularly differentiated populations; even Cantalupensis and Reticulatus, which have been merged by some authors because share many morphological features (Munger and Robinson, 1991; Robinson and Decker-Walters, 1997). However, many cultivars with morphological and molecular intermediate features exist among these three groups. Within ssp. agrestis, Far Eastern melons, Conomon, Makuwa, and Chinensis, are genetically quite close despite their differential morphological features, but they can be molecularly differentiated from other groups of this subspecies, such as the Indian Acidulus and the African Tibish (Blanca et al., 2012; Esteras et al., 2013; Leida et al., 2015).

In 2012, Brazil was the ninth largest melon producer in the world with an output of 575,000 t (FAO, 2014). Brazilian melon production is mostly $(>95 \%)$ concentrated in the northeastern region, mainly in the states of Ceará and Rio Grande do Norte (Nunes et al., 2011). Melons are grown in large fields (15 to $20 \mathrm{ha}$ ) and almost all of the production is exported to Europe and the United States. Brazilian commercial production is mainly based on hybrid cultivars belonging to groups Inodorus (casaba melons of the market classes Amarillo and Piel de Sapo) and Cantalupensis (market classes Charentais, muskmelons, and Galia) (Nunes et al., 2005).

Melon, like other cucurbits, such as watermelon [Citrullus lanatus (Romão, 2000)] and gherkin [Cucumis anguria (Queiróz, 2004)], is supposed to have been introduced in America by African slaves. In addition, independent introductions may have occurred via European immigrants, as some types of melon landraces are called "portuguese melons" by farmers in southern Brazil. There is a large set of traditional cultivars adapted to different soil and climatic conditions that are grown under traditional cultural systems in different regions of the country. Since their introduction in Brazil, melon materials have undergone an intense process of selection and crossing by small farmers. Regardless, Brazil has not been considered to be a center of melon diversification.

Efforts have been made to collect these landraces in various states, mainly in northeastern Brazil, where they are still cultivated under subsistence agriculture conditions (Delwing et al., 2007). However, because not all northeastern Brazilian states have been sampled in previous collections, further collection efforts are necessary to access a complete representation of the extant Brazilian landraces statuses. In addition, only a few reports (Aragão et al., 2013; Neitzke et al., 2009) have dealt with the morphological and molecular characterization of Brazilian melon landraces, and none have provided data on comparisons between these landraces and reference germplasms of diverse origins to help trace the origins of Brazilian melon landraces.

The purpose of this research was to study the diversity of Brazilian melon landraces and their relationships with melon genotypes of various origins. To accomplish this, we performed morphological and molecular analyses using a set of simple sequence repeat (SSR) markers and a collection of 40 melon landraces from different northeastern Brazilian states, including some that were not previously sampled, and compared them with a reference melon accession set.

\section{Materials and Methods}

Plant material. In this study, we analyzed 61 melon accessions. Forty accessions were Brazilian landraces collected from nine states in northeastern Brazil (Fig. 1; Table 1). Fruit of selected single plants, representing all of the fruit types

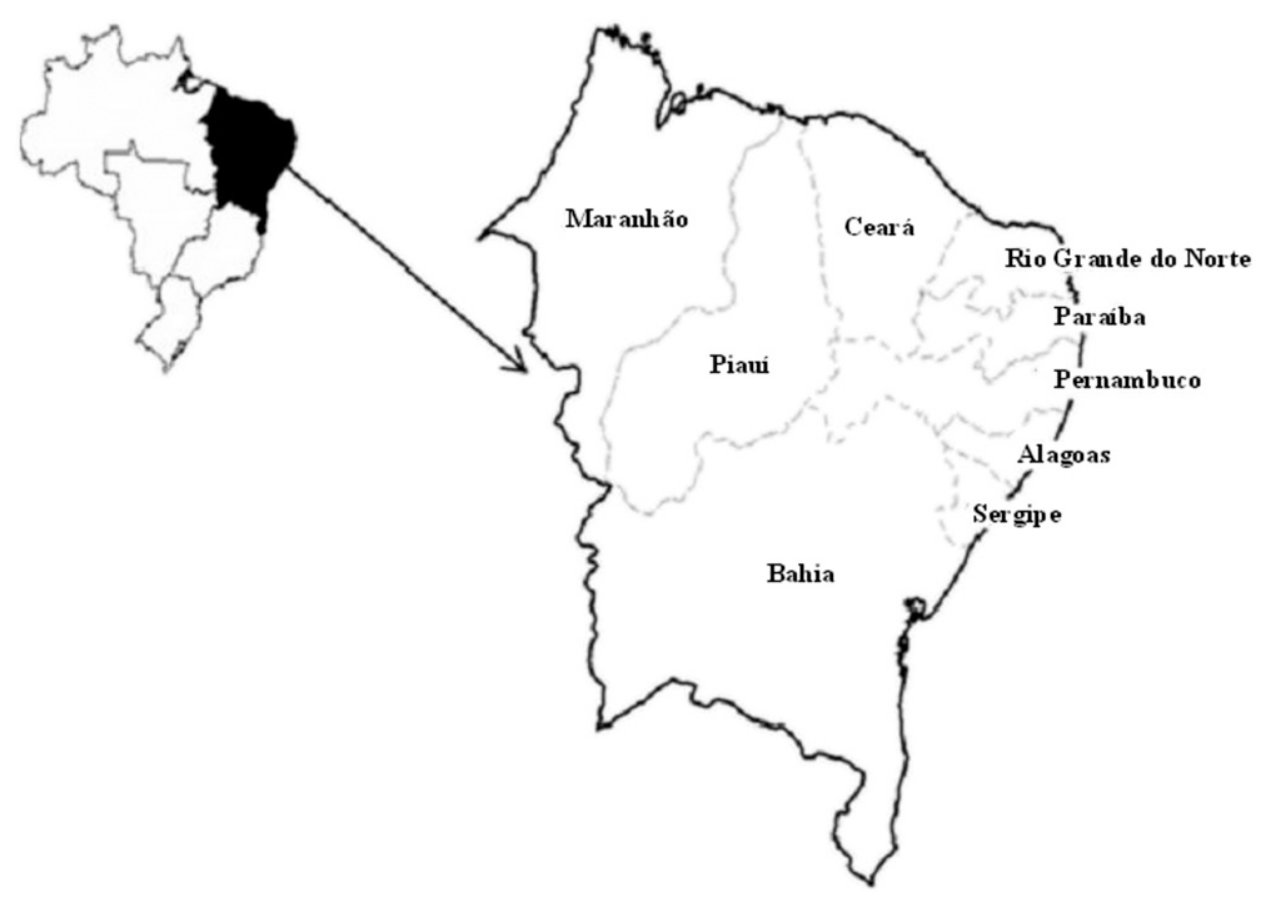

Fig. 1. Map of northeastern Brazil showing the nine states where 40 Brazilian melon landraces were collected. 
cultivated in each surveyed area, were collected. Additional 21 accessions were included as references (Table 2). These references represent the main horticultural groups of the species: Inodorus, Cantalupensis, Reticulatus, Ameri, Flexuosus, Dudaim, Momordica, Makuwa, Chinensis, Tibish, Acidulus, Chito, and wild agrestis (represented by two wild accessions, one from Africa and one from India). All of the accessions used as references were previously morphologically characterized, along with a larger collection of melons, to confirm their classification in the corresponding horticultural group (Leida et al., 2015).

Morphological evaluation. We evaluated 40 Brazilian landraces (Table 1; Fig. 2) and three commercial controls (Table 2) for yield and morphological traits in 2013 at the Federal Rural University of Semiarid, Mossoró, Rio Grande do Norte State, Brazil. Accessions were sown in commercial substrate (Polifertil ${ }^{\circledR}$, Uberaba, Brazil) and seedlings (at the three-true-leaf stage) were transplanted to the field. The experiment was carried out in a randomized complete block design with three replications of each accession, each replication with 10 plants. Betweenrow and within-row spacings were 2.0 and $0.30 \mathrm{~m}$, respectively. Plants were furrow irrigated and fertilized according to standard cultural practices. The eight central plants of each replication were used for vine characterization and fruit sampling. One fruit per plant was harvested at the fully mature stage from all eight plants of each replication, and a total of 24 fruit were characterized per accession. The harvesting of fruit was done over a $15-\mathrm{d}$ period. The formation of an abscission layer was used as an indicator of maturity in climacteric accessions.

Qualitative and quantitative morphological traits were recorded according to the descriptor of the International Plant Genetic Resources Institute (2003). The qualitative traits were fruit shape, mature fruit rind and flesh color, occurrence of fruit cracking, and sex expression. The classical markers of climacteric ripening, that is, the formation of an abscission layer and fruit detachment,
Table 1. Inventory of 40 melon landraces collected from nine states in northeastern Brazil.

\begin{tabular}{|c|c|c|c|c|c|}
\hline$\overline{\text { No. }}$ & Accession & State & No. & Accession & State \\
\hline 1 & MA-1 & Maranhão & 21 & $\mathrm{RN}-8$ & Rio Grande do Norte \\
\hline 2 & MA-2 & Maranhão & 22 & RN-9 & Rio Grande do Norte \\
\hline 3 & MA-3 & Maranhão & 23 & PB-1 & Paraíba \\
\hline 4 & MA-4 & Maranhão & 24 & PB-2 & Paraíba \\
\hline 5 & MA-5 & Maranhão & 25 & PB-3 & Paraíba \\
\hline 6 & MA-6 & Maranhão & 26 & PB-4 & Paraíba \\
\hline 7 & PI-1 & Piauí & 27 & PE-1 & Pernambuco \\
\hline 8 & PI-2 & Piauí & 28 & PE-2 & Pernambuco \\
\hline 9 & PI-3 & Piauí & 29 & PE-3 & Pernambuco \\
\hline 10 & CE-1 & Ceará & 30 & PE-4 & Pernambuco \\
\hline 11 & CE-2 & Ceará & 31 & AL-1 & Alagoas \\
\hline 12 & CE-3 & Ceará & 32 & AL-2 & Alagoas \\
\hline 13 & CE-4 & Ceará & 33 & AL-3 & Alagoas \\
\hline 14 & $\mathrm{RN}-1$ & Rio Grande do Norte & 34 & SE-1 & Sergipe \\
\hline 15 & $\mathrm{RN}-2$ & Rio Grande do Norte & 35 & SE-2 & Sergipe \\
\hline 16 & $\mathrm{RN}-3$ & Rio Grande do Norte & 36 & BA-1 & Bahia \\
\hline 17 & $\mathrm{RN}-4$ & Rio Grande do Norte & 37 & BA-2 & Bahia \\
\hline 18 & RN-5 & Rio Grande do Norte & 38 & BA-3 & Bahia \\
\hline 19 & $\mathrm{RN}-6$ & Rio Grande do Norte & 39 & BA-4 & Bahia \\
\hline 20 & RN-7 & Rio Grande do Norte & 40 & BA-5 & Bahia \\
\hline
\end{tabular}

Table 2. Inventory of 21 melon accessions used as references for morphological and molecular analyses. $^{z}$

\begin{tabular}{lcll}
\hline Accession & \multicolumn{1}{c}{ Code } & \multicolumn{1}{c}{ Origin } & \multicolumn{1}{c}{ Group } \\
\hline & Cucumis melo ssp. melo & & \\
'Amaral'y & In-01 & Rijk Zwaan ${ }^{\circledR}$ & Inodorus \\
'Mandacaru'y & In-02 & Fitó $^{\circledR}$ & Inodorus \\
'Natal'y & In-03 & Rijk Zwaan ${ }^{\circledR}$ & Inodorus \\
'Piñonet Piel de sapo' & In-PS & Spain & Inodorus \\
'Honeydew' & In-HD & USA & Inodorus \\
'Kirkagac' & In-KIR & Turkey & Inodorus \\
'Véndrantais' & Can-VED & France & Cantalupensis \\
'Dulce' & Ret-DUL & USA & Reticulatus \\
'Kizil Uruk' & Am-KU & Uzbequistan & Ameri \\
Snakemelon & Flex-SNK & Arabia & Flexuosus \\
'Queen's Pocket Melon'x & Dud-QPM & Afghanistan & Dudaim
\end{tabular}

\begin{tabular}{llll} 
'Kharbuja' & Mom-KHA & India & Momordica \\
PI 124112w & Mom-PI124 & India & Momordica \\
MR-1 & Mom-MR1 & India & Momordica \\
'Ginsen Makuwa' & Mak-GM & Japan & Makuwa \\
PI 161375 ('SongWhan-Charmi') & Chin-SC & Korea & Chinensis \\
Tibish & Tib-TIB & Sudan & Tibish \\
PI 482420 (TGR 1551) & Ac-TGR & Zimbabwe & Acidulus \\
PI $164320($ 'Velleri') & Chi-VELL & India & Chito \\
PI 185111 (15591) & Ag-15591 & Ghana & Wild agrestis \\
Wild Chibbar & Ag-WCh & India & Wild agrestis \\
\hline
\end{tabular}

${ }^{\mathrm{z}}$ This collection was established on the framework of a previous project MELRIP 2007-2010 (Esteras et al., 2013), and was multiplied by the Institute for the Conservation and Breeding of the Agrobiodiversity (COMAV) Cucurbits Breeding Group and is currently conserved the COMAV genebank. All the accessions were morphologically characterized previously, along with a larger collection of melons, to confirm their classification in the corresponding horticultural group (Leida et al., 2015).

${ }^{\mathrm{y}}$ Commercial genotypes evaluated in morphological analysis.

${ }^{\mathrm{x}}$ Genotypes kindly provided by M. Pitrat.

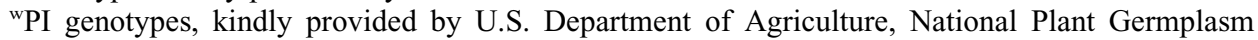
System. 


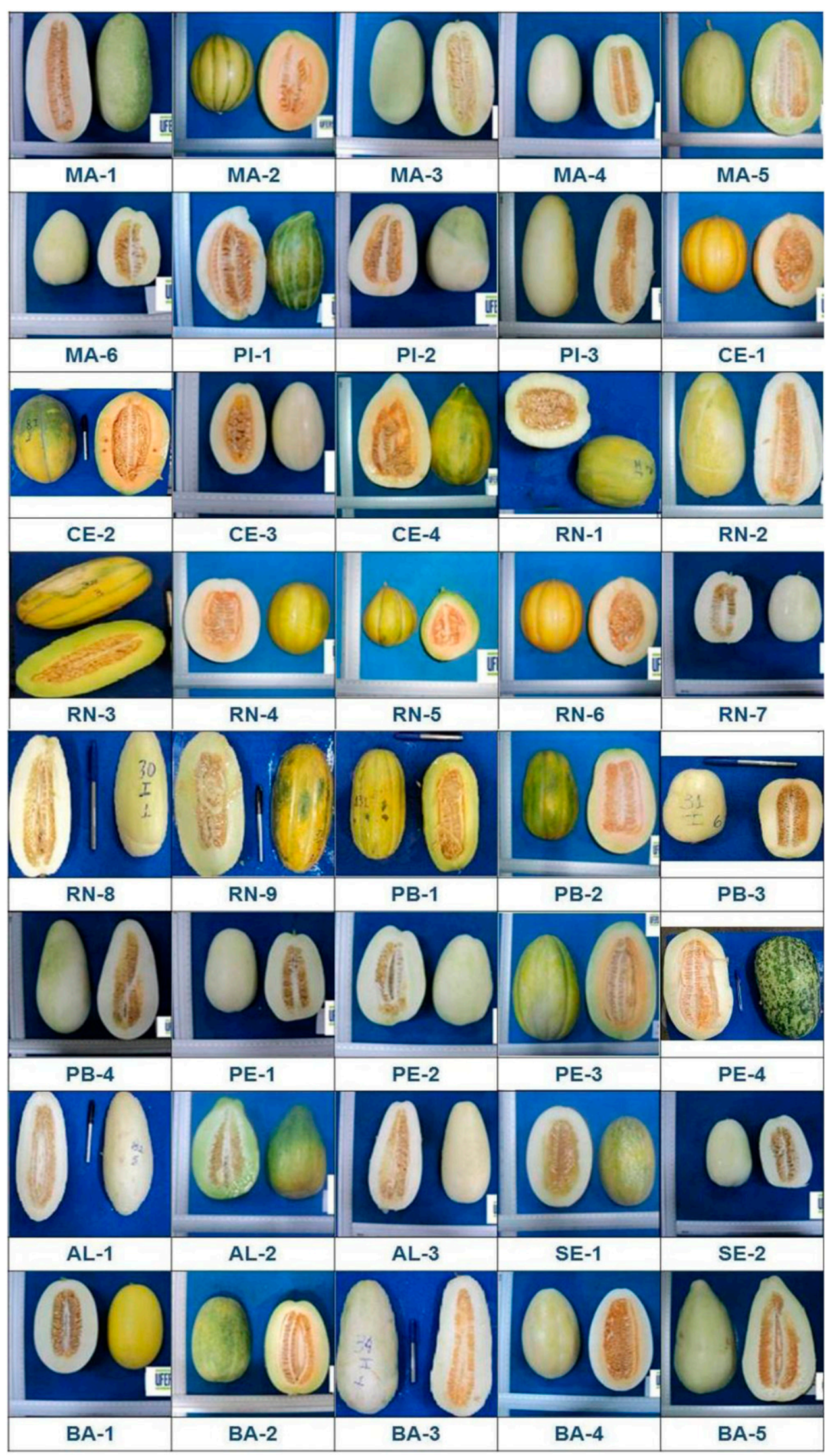

Fig. 2. Fruit of 40 Brazilian melon landraces collected in northeastern states. were used to classify the accessions as climacteric or nonclimacteric. The quantitative traits were 100seed weight, days from transplanting ( 2 weeks after seed sowing) to the opening of the first female flower, days from pollination to fruit maturity, fruit weight, internal cavity diameter, flesh thickness, flesh firmness [measured with a digital penetrometer (FHT-803 ${ }^{\circledR}$; General Tools, Melrose, MA)], total soluble solids [TSS measured with a digital refractometer (Atago ${ }^{\circledR}$; Atago, Tokyo, Japan)], $\mathrm{pH}$ [measured with a pH meter (Digimed ${ }^{\circledR}$ DM-22; Digimed Analytical Instrumentation, Beijing, China)], titratable acidity (quantified through titration of a fruit juice sample with $0.05 \mathrm{~N}$ $\mathrm{NaOH}$, with phenolphthalein as indicator), and ascorbic acid [measured according to the process described by Dhillon et al. (2009)].

Apart from the 24 fruits that were characterized per accession (one per plant), all of the fruit that reached full maturity in each plant were collected and used to measure yield. Two parameters were recorded for each accession: the number of fruit per plant (calculated dividing the total number of fruit harvested in all the plants of each replication by the plant number, usually 8) and the yield [calculated weighing all of the fruit harvested in all of the plants of each replication (spacing $2.0 \times 0.30 \mathrm{~m}$ ), expressed in tonnes per hectare].

DNA extraction. Genomic DNA was extracted from young leaf tissues sampled from the eight plants of each replication at the three-trueleaf stage using the cetyl trimethylammonium bromide procedure as described by Doyle and Doyle (1990) and modified by Esteras et al. (2013). Subsequently, all DNA was quantified by a spectrophotometer (Nanodrop ${ }^{\circledR}$ 2000; Thermo Fisher Scientific, Wilmington, DE), which determines the concentration and purity of DNA. After quantification, the DNA of the different plants of each accession was bulked and diluted to a $10 \mathrm{ng} \cdot \mathrm{mL}^{-1}$ concentration, which is appropriate for polymerase chain reaction (PCR).

Microsatellite analysis. We used in this study 13 SSR markers. These markers were generated from 
a melon genomic library (Chiba et al., 2003) and were previously checked for polymorphisms using cultivars belonging to six melon botanical varieties. They are distributed in the melon genome. Their physical position in the last version of the melon genome v3.5 (Melonomics Project Team, 2014) and an estimate of the genetic position, according to the genetic map in melonomics (Garcia-Mas et al., 2012), are included in Supplemental Table 1.

PCR reactions were performed in a final volume of $10 \mu \mathrm{L}$, as follows: $0.25 \mathrm{~mm}$ dNTP, $0.3 \mathrm{~mm}$ of each primer, PCR buffer (10 mm Tris-HCl, pH 8.3), $2.5 \mu \mathrm{M} \mathrm{MgCl} 2,0.5 \mathrm{U}$ Taq DNA polymerase, $10 \mathrm{ng}$ genomic DNA, and sterile ultrapure water. The amplification program steps were as follows: initial DNA denaturation for $2 \mathrm{~min}$ at $94{ }^{\circ} \mathrm{C}$ followed by 40 cycles of DNA denaturation for $1 \mathrm{~min}$ at $94{ }^{\circ} \mathrm{C}$, annealing for $1 \mathrm{~min}$ at 50 to $58^{\circ} \mathrm{C}$ (depending on the primer) and amplification for $1 \mathrm{~min}$ at $72^{\circ} \mathrm{C}$, then a final DNA amplification for $7 \mathrm{~min}$ at $72{ }^{\circ} \mathrm{C}$. The PCR was performed in a thermocycler (PTC-100 ${ }^{\circledR}$; MJ Research, Hayward, CA). The products of amplification were subjected to $6 \%$ polyacrylamide gels containing 7.0 $\mathrm{M}$ urea in Tris-borate-EDTA acid buffer. Electrophoresis was performed at $45 \mathrm{~W}$ of constant power, and gels were stained with silver nitrate. Molecular data were obtained from the visualization of bands, considering the polymorphic markers and their respective alleles, which were determined by the presence/absence of bands and sizes of DNA fragments at each locus. Information about allele sizes in each genotype is included in Supplemental Table 2. All of the analyses were performed at the Laboratory of Molecular Biology/Embrapa Algodão, Campina Grande, Paraíba State, Brazil.

Statistical analysis. The mean values of the three replications (of eight fruit each), the standard deviations of the means, and the range were calculated for each trait. All quantitative data were subjected to analysis of variance. The contribution of each morphological trait to the overall variation was evaluated with principal component analysis (PCA) with the software PROC IML (SAS version 9.2; SAS Institute, Cary, NC). The number of alleles, the major allele frequency, the gene diversity, the heterozygosity and the PIC according to Nei et al. (1983) were calculated with Powermarker software (Liu and Muse, 2005). A factor correspondence analysis
(FCA) was performed with NTSYSpc 2.11W (Exeter Software, Setauket, NY).

\section{Results}

MORPHOLOGICAL CHARACTERIZATION AND FIELD OBSERVATIONS. Out of the 40 Brazilian accessions, only four developed round fruit; the others were oblate, elongated, elliptical, and pyriform (Table 3; Fig. 2). The fruit exhibited two rind colors at maturity: cream $(62.5 \%)$ and yellow $(37.5 \%)$; there were three different flesh colors: white $(72.5 \%)$, orange $(17.5 \%)$, and green $(10.0 \%)$. Most of the accessions (57.5\%) suffered fruit cracking at maturity. All of the Brazilian accessions were monoecious.

Table 3. Qualitative traits scored in 40 melon landraces collected from nine states in northeastern Brazil and in three commercial hybrids.

\begin{tabular}{|c|c|c|c|c|c|}
\hline \multirow[b]{2}{*}{ Accession } & \multirow[b]{2}{*}{ Fruit shape } & \multicolumn{2}{|c|}{ Mature fruit } & \multirow{2}{*}{$\begin{array}{l}\text { Occurrence of } \\
\text { fruit cracking }\end{array}$} & \multirow[b]{2}{*}{ Sex expression } \\
\hline & & Rind color & Flesh color & & \\
\hline$\overline{\mathrm{MA}-1}$ & Elongated & Cream & White & Yes & Monoecious \\
\hline MA-2 & Round & Yellow & Orange & No & Monoecious \\
\hline MA-3 & Elliptical & Cream & White & Yes & Monoecious \\
\hline MA-4 & Elliptical & Cream & White & Yes & Monoecious \\
\hline MA-5 & Elliptical & Cream & Green & No & Monoecious \\
\hline MA-6 & Oblate & Cream & White & Yes & Monoecious \\
\hline PI-1 & Oblate & Cream & White & Yes & Monoecious \\
\hline PI-2 & Oblate & Cream & White & Yes & Monoecious \\
\hline PI-3 & Oblate & Cream & White & Yes & Monoecious \\
\hline CE-1 & Round & Yellow & White & No & Monoecious \\
\hline CE-2 & Oblate & Yellow & Orange & No & Monoecious \\
\hline CE-3 & Oblate & Cream & White & Yes & Monoecious \\
\hline CE-4 & Pyriform & Yellow & Orange & No & Monoecious \\
\hline RN-1 & Round & Yellow & White & No & Monoecious \\
\hline $\mathrm{RN}-2$ & Elongated & Cream & White & Yes & Monoecious \\
\hline $\mathrm{RN}-3$ & Elongated & Yellow & Green & No & Monoecious \\
\hline $\mathrm{RN}-4$ & Round & Yellow & White & No & Monoecious \\
\hline $\mathrm{RN}-5$ & Elongated & Yellow & Orange & Yes & Monoecious \\
\hline RN-6 & Oblate & Yellow & White & No & Monoecious \\
\hline $\mathrm{RN}-7$ & Oblate & Cream & White & Yes & Monoecious \\
\hline $\mathrm{RN}-8$ & Elongated & Cream & White & Yes & Monoecious \\
\hline $\mathrm{RN}-9$ & Elongated & Yellow & Green & No & Monoecious \\
\hline PB-1 & Oblate & Yellow & Orange & No & Monoecious \\
\hline PB-2 & Oblate & Yellow & Orange & No & Monoecious \\
\hline PB-3 & Oblate & Cream & White & Yes & Monoecious \\
\hline PB-4 & Elongated & Cream & White & Yes & Monoecious \\
\hline PE-1 & Oblate & Cream & White & No & Monoecious \\
\hline PE-2 & Oblate & Cream & White & No & Monoecious \\
\hline PE-3 & Elliptical & Yellow & Orange & No & Monoecious \\
\hline PE-4 & Elongated & Cream & White & Yes & Monoecious \\
\hline AL-1 & Elongated & Cream & White & Yes & Monoecious \\
\hline AL-2 & Oblate & Yellow & Green & No & Monoecious \\
\hline AL-3 & Elongated & Cream & White & Yes & Monoecious \\
\hline SE-1 & Elliptical & Cream & White & Yes & Monoecious \\
\hline SE-2 & Oblate & Cream & White & Yes & Monoecious \\
\hline BA-1 & Elliptical & Yellow & White & No & Monoecious \\
\hline BA-2 & Oblate & Cream & White & Yes & Monoecious \\
\hline BA-3 & Elongated & Cream & White & Yes & Monoecious \\
\hline BA-4 & Elliptical & Cream & White & Yes & Monoecious \\
\hline BA-5 & Oblate & Cream & White & Yes & Monoecious \\
\hline In-01 & Round & Yellow & White & No & Andromonoecious \\
\hline In-02 & Round & Yellow & White & No & Andromonoecious \\
\hline In-03 & Round & Yellow & White & No & Andromonoecious \\
\hline
\end{tabular}


The accessions varied in characteristics related to seed weight and time of flowering and fruit maturity (Table 4). Mean seed weights ranged from 0.9 to $4.8 \mathrm{~g}$, and in many accessions, the seeds were smaller than those in the Inodorus commercial cultivars, in which seed weight ranged from 3.1 to $4.8 \mathrm{~g}$. Most accessions were more late flowering than the controls. Days to first female flower ranged from 23 to $30.5 \mathrm{~d}$ after transplanting (DAT), and the fruit ripening period ranged from 28 to $42.5 \mathrm{~d}$ after pollination (DAP), while Inodorus cultivars flowered at 23 DAT and developed mature fruit at 28 to 29 DAP.

The Brazilian accessions also exhibited high variability in the quantitative fruit traits (weight, diameter of the internal cavity, flesh thickness, flesh firmness, number of fruit per plant, and yield) (Tables 4 and 5). Most of them exhibited a climacteric ripening behavior, forming a clear abscission layer that resulted in fruit slip. Accordingly, due to this climacteric behavior, most of them had lower fruit flesh firmness (10.3 to $32.6 \mathrm{~N})$ than the non-climacteric Inodorus commercial cultivars (28.1 to $33.4 \mathrm{~N}$ ). The only nonclimacteric Brazilian accession was BA-1, with 44.9 $\mathrm{N}$ flesh firmness.

All Brazilian accessions had fruit with low to medium sugar content (TSS from $3.1 \%$ to $7.6 \%$ ) (Table 6 ) and none reached the level of soluble solids found in the Inodorus sweet melons ( $9.0 \%$ to $9.2 \%)$. Overall accessions (controls included), $\mathrm{pH}$ ranged from 3.0 to 7.2 , titratable acidity from $0.05 \%$ to $0.59 \%$, and ascorbic acid content from 3.62 to $9.11 \mathrm{mg} / 100 \mathrm{~g}$ of fruit flesh. In some Brazilian accessions, mature fruit were quite acidic, and accordingly, they had high titratable acid and ascorbic acid contents (Table 6).

The main traits that contribute to the morphological variation among the landraces (PCA) are shown in Fig. 3A. The estimates of the correlations between the traits ranged from low to medium magnitude ( $r=$ 0.3 to 0.56$)$. Therefore, all variables were retained in the PCA. The first principal component, which accounted for $30.13 \%$ of the total variation, mainly grouped the accessions according to seed and fruit
Table 4. Mean values (of three replications of eight fruits each), standard deviation of the mean, and range of five melon seed, flower, and fruit traits of 40 Brazilian melon landraces collected from nine states in northeastern and three commercial hybrids.

\begin{tabular}{|c|c|c|c|c|c|c|c|c|}
\hline \multirow[b]{2}{*}{ Accession } & \multicolumn{2}{|l|}{$\mathrm{SW}^{\mathrm{z}}$} & \multicolumn{2}{|c|}{$\mathrm{DFF}^{\mathrm{y}}$} & \multicolumn{2}{|c|}{$\mathrm{DFM}^{\mathrm{x}}$} & \multirow{2}{*}{$\frac{\mathrm{FN}^{\mathrm{w}}}{\mathrm{Mean} \pm \mathrm{SD}}$} & \multirow{2}{*}{$\frac{\mathrm{YID}^{\mathrm{v}}}{\text { Mean } \pm \mathrm{SD}}$} \\
\hline & Mean \pm SD & Range & Mean \pm SD & Range & Mean \pm SD & Range & & \\
\hline AA-1 & $2.6 \pm 0.8$ & $1.8-2.8$ & $23.0 \pm 0.5$ & $21-24$ & $34.5 \pm 0.7$ & $30-35$ & $1.8 \pm 0.4$ & $4.7 \pm 10.9$ \\
\hline A-2 & 0.9 & $3.0-$ & $27.0 \pm 1.0$ & $25-28$ & $.0 \pm 0.8$ & & 0.5 & $20.1 \pm 15.5$ \\
\hline IA-3 & $8 \pm 0.3$ & $4.0-4.9$ & $28.0 \pm 1.3$ & $26-29$ & $39.5 \pm 1.5$ & $37-40$ & $1.7 \pm 0.5$ & $31.7 \pm 8.9$ \\
\hline A-4 & $2.4 \pm 0.9$ & $2.0-2.6$ & $27.0 \pm 0.5$ & $26-28$ & $38.5 \pm 1.6$ & $36-41$ & $1.8 \pm 0.3$ & $38.9 \pm 6.7$ \\
\hline & $4.0 \pm 0.1$ & $3.8-4.1$ & $26.5 \pm 0.5$ & $25-28$ & $38.5 \pm 1.0$ & $36-39$ & $1.1 \pm 0.5$ & $25.2 \pm 5.1$ \\
\hline A-6 & $1.7 \pm 0.1$ & $1.5-2.0$ & $29.0 \pm 0.5$ & $27-29$ & $38.5 \pm 1.5$ & $36-41$ & $2.1 \pm 2.0$ & $7.00 \pm 4.1$ \\
\hline & 0.2 & $1.6-2.3$ & $28.5 \pm 0.6$ & $26-30$ & $38.0 \pm 0.5$ & $36-$ & $2.0 \pm 1.0$ & $24.7 \pm 8.3$ \\
\hline-2 & $2.2 \pm 1.0$ & $1.6-2.5$ & $27.5 \pm 3.8$ & $26-29$ & $39.0 \pm 1.0$ & $33-40$ & $2.4 \pm 0.5$ & $31.1 \pm 3.6$ \\
\hline & 0.1 & $2.0-2.6$ & \pm 2.0 & $22-26$ & \pm 1.0 & & $1.9 \pm 0.7$ & $37.7 \pm 2.1$ \\
\hline-1 & $9 \pm 0.1$ & $3.4-4.0$ & $24.5 \pm 1.0$ & $22-25$ & $35.5 \pm 1.4$ & $32-38$ & $1.2 \pm 0.5$ & $25.6 \pm 3.6$ \\
\hline & 0.1 & $3.3-4.1$ & $24.5 \pm 0.5$ & $23-26$ & \pm 0.7 & & 0.8 & $4.2 \pm 3.1$ \\
\hline-3 & 0.6 & $2.4-1.9$ & $30.0 \pm 3.0$ & $28-31$ & $39.0 \pm 1.3$ & $34-42$ & $1.9 \pm 2.5$ & $21.4 \pm 5.8$ \\
\hline-4 & 0.5 & $3.5-4.0$ & $23.0 \pm 0.9$ & $22-24$ & $36.0 \pm 0.5$ & $33-37$ & $1.4 \pm 0.8$ & $44.3 \pm 8.9$ \\
\hline N-1 & 0.7 & $2.3-2.8$ & $27.5 \pm 2.1$ & $25-29$ & \pm 0.7 & $33-38$ & $2.0 \pm 0.5$ & $19.9 \pm 8.9$ \\
\hline & 0.2 & 1.3 & $28.0 \pm 2.3$ & $26-29$ & \pm 1.0 & $38-41$ & $2.5 \pm 1.0$ & $45.8 \pm 9.8$ \\
\hline & 0.1 & $1.9-3.3$ & \pm 0.8 & $22-26$ & \pm 0.6 & & $1.1 \pm$ & $22.5 \pm 9.3$ \\
\hline-4 & 0.1 & $3.6-3.9$ & $26.5 \pm 0.7$ & $23-29$ & $37.0 \pm 1.2$ & $33-$ & $1.0 \pm 0.8$ & $37.8 \pm 7.3$ \\
\hline & 0.3 & $2.4-3.0$ & $27.5 \pm 0.9$ & 24-29 & \pm 1.4 & & $1.3 \pm 0.6$ & $21.8 \pm 7.9$ \\
\hline 6 & 0.1 & $4.0-4.5$ & $27.5 \pm 1.3$ & $25-29$ & \pm 0.7 & 33 & $1.1 \pm$ & $25.8 \pm 4.7$ \\
\hline & 0.1 & & $24.5 \pm 1.5$ & $23-28$ & \pm 0.5 & & $2.8 \pm$ & $15.2 \pm 4.7$ \\
\hline I-8 & 0.3 & $3.0-3.5$ & $28.5 \pm 2.0$ & 24-29 & \pm 2.0 & $34-40$ & $1.6 \pm$ & $22.7 \pm 1.0$ \\
\hline & 0.1 & $3.0-$ & $26.5 \pm 0.4$ & $23-27$ & 0.9 & & $1.2 \pm$ & $0.5 \pm 5.2$ \\
\hline $3-1$ & 0.2 & $3.2-4.1$ & $29.0 \pm 1.5$ & $27-30$ & \pm 0.8 & 38 & $1.1 \pm$ & $18.1 \pm 4.7$ \\
\hline 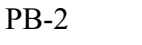 & 0.3 & $4.0-4.6$ & \pm 4.0 & $23-26$ & \pm 3.0 & & $1.3 \pm$ & $24.1 \pm 4.5$ \\
\hline $3-3$ & 0.1 & $1.5-2.0$ & $29.0 \pm 2.2$ & $25-29$ & \pm 1.5 & $36-40$ & $2.3 \pm$ & $16.8 \pm 5.7$ \\
\hline$D-4$ & 0.1 & $1.0-1.6$ & \pm 1.0 & $26-31$ & \pm 0.6 & 38 & $1.9 \pm 0.8$ & $12.1 \pm 4.1$ \\
\hline$E-1$ & 0.1 & $1.1-1.6$ & \pm 0.3 & $27-31$ & \pm 0.6 & & $2.1 \pm 1.5$ & $18.0 \pm 9.1$ \\
\hline-2 & 0.2 & 0.9 & $29.5 \pm 2.0$ & $27-30$ & \pm 0.8 & & $2.0 \pm$ & $19.4 \pm 4.7$ \\
\hline-3 & 0.4 & $4.0-4.6$ & $29.0 \pm 0.8$ & $26-29$ & \pm 0.5 & 36 & $1.3 \pm 1.0$ & $13.1 \pm 4.2$ \\
\hline E-4 & 1.6 & $2.6-3.1$ & $23.5 \pm 1.7$ & $22-29$ & \pm 2.4 & $37-40$ & $1.1 \pm 1.0$ & $16.1 \pm 8.5$ \\
\hline L-1 & \pm 0.7 & $2.4-2.9$ & $28.0 \pm 1.2$ & 24-29 & \pm 0.5 & $37-40$ & $1.9 \pm 1.2$ & $42.5 \pm 5.7$ \\
\hline L-2 & 0.1 & $2.6-2.9$ & \pm 1.3 & $23-27$ & \pm 1.0 & & $1.3 \pm$ & $17.1 \pm 11.9$ \\
\hline L-3 & $=0.4$ & $2.7-3.2$ & $27.5 \pm 1.0$ & $25-27$ & \pm 1.5 & 34 & $1.3 \pm$ & $26.0 \pm 5.2$ \\
\hline E-1 & $0 \pm 0.1$ & $1.5-2.1$ & $26.5 \pm 0.4$ & $23-27$ & \pm 3.5 & $34-41$ & $1.5 \pm 0.6$ & $17.0 \pm 8.8$ \\
\hline$L-2$ & $0 \pm 0.5$ & $1.4-2.2$ & $30.0 \pm 0.8$ & $28-31$ & \pm 2.0 & $37-$ & $3.1 \pm 1.5$ & $21.7 \pm 5.2$ \\
\hline A-1 & $9 \pm 0.1$ & $0.7-1.1$ & $30.0 \pm 0.5$ & $28-32$ & \pm 0.7 & & $3.9 \pm 2.0$ & $17.4 \pm 5.9$ \\
\hline & $=0.1$ & $1.6-1.9$ & $25.5 \pm 2.5$ & $23-30$ & \pm 0.6 & & $1.5 \pm 0.9$ & $19.9 \pm 3.9$ \\
\hline BA-3 & $2.9 \pm 0.1$ & $2.7-3.1$ & $27.0 \pm 1.0$ & $25-28$ & $40.5 \pm 0.5$ & $37-41$ & $1.4 \pm 1.5$ & $37.1 \pm 8.1$ \\
\hline A-4 & $3 \pm 0.1$ & $2.5-2.9$ & $26.5 \pm 0.6$ & $24-28$ & $37.0 \pm 2.0$ & $36-39$ & $1.9 \pm 0.9$ & $33.0 \pm 9.3$ \\
\hline BA-5 & $2.5 \pm 0.2$ & $2.0-2.7$ & $30.5 \pm 1.2$ & $28-32$ & $40.5 \pm 1.0$ & $37-41$ & $2.4 \pm 1.0$ & $34.7 \pm 9.6$ \\
\hline $\mathrm{n}-01$ & $4.0 \pm 0.1$ & $3.8-4.4$ & $23.0 \pm 1.1$ & $21-24$ & $29.0 \pm 0.5$ & $27-30$ & $1.1 \pm 1.0$ & $15.3 \pm 5.7$ \\
\hline In-02 & $3.1 \pm 0.1$ & $2.9-3.9$ & $23.0 \pm 1.3$ & $20-24$ & $28.0 \pm 2.0$ & $27-30$ & $1.6 \pm 1.0$ & $26.8 \pm 7.8$ \\
\hline In-03 & $4.8 \pm 0.4$ & $4.0-4.9$ & $23.0 \pm 1.1$ & $22-26$ & $29.0 \pm 2.5$ & $27-30$ & $1.2 \pm 1.0$ & $12.7 \pm 5.9$ \\
\hline$F^{\mathrm{u}}$ & 7.9 & & 4.6 & & 9.3 & & 3.8 & 2.2 \\
\hline SE & 0.4 & & 1.1 & & 0.9 & & 0.7 & 6.8 \\
\hline LSD & 0.6 & & 1.9 & & 1.6 & & 1.2 & 11.4 \\
\hline
\end{tabular}

${ }^{\mathrm{z}} 100$-seed weight $(\mathrm{g} / 100$ seed).

${ }^{\mathrm{y}}$ Days from transplanting ( 2 weeks after seed sowing) to the opening of the first female flower. ${ }^{x}$ Days from pollination to fruit maturity.

${ }^{\text {w}}$ Fruit number; calculated per plant dividing the total number of fruit harvested in all the plants of each replication by the plant number.

${ }^{v}$ Yield $\left(\mathrm{t} \cdot \mathrm{ha}^{-1}\right)$; calculated weighting all the fruit harvested in all the plants of each replication (spacing $2.0 \times 0.30 \mathrm{~m}$ ).

${ }^{\text {u }} F=$ estimated by Snedecor's $F$ test.

tLeast significant difference by $t$ test. 
Table 5. Mean values (of three replications of eight fruit each), standard deviation of the mean, and range of four melon fruit traits of 40 Brazilian melon landraces collected from nine states in northeastern and three commercial hybrids.

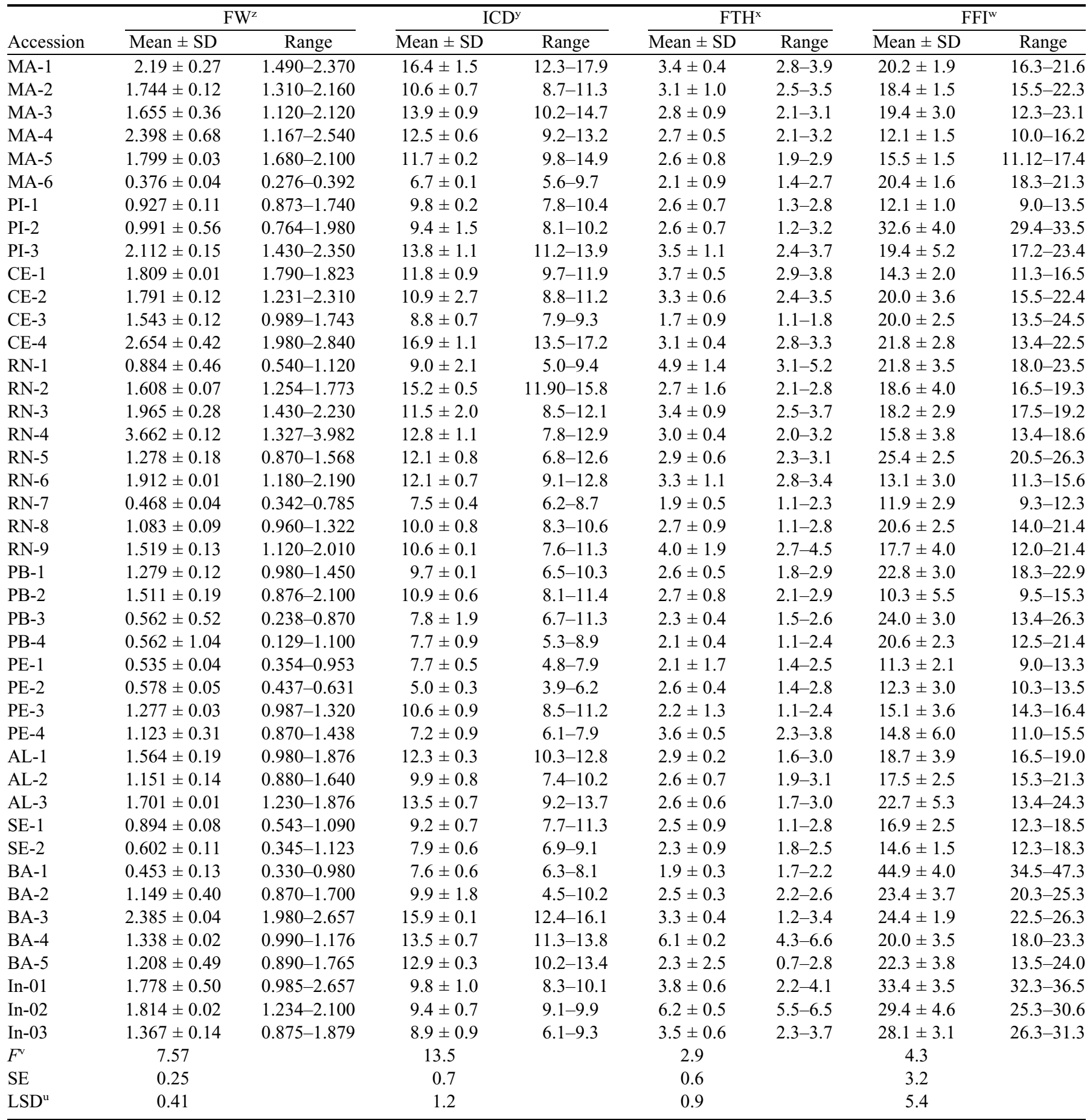

${ }^{\mathrm{z}}$ Fruit weight $(\mathrm{kg})$.

yInternal cavity diameter $(\mathrm{cm})$.

${ }^{x}$ Flesh thickness $(\mathrm{cm})$.

${ }^{\mathrm{w}}$ Flesh firmness $\left(\mathrm{N} \cdot \mathrm{m}^{-2}\right)$; measured with a digital penetrometer.

${ }^{\text {v }} F=$ estimated by Snedecor's $F$ test.

"Least significant difference by $t$ test.

weights and flowering time (Fig. 3A). This component separated a group of accessions (group I in Fig. 3B), which is characterized by delayed flowering (mean \pm SE) $(28.2 \pm 0.53$ DAT) and, in general, smaller seeds and fruit (seed and fruit weight means of
$2.1 \pm 0.21 \mathrm{~g}$ and $0.756 \pm 0.100 \mathrm{~kg}$, respectively) with sweeter flesh (TSS 5.9\% $\pm 0.18 \%$ ) than in most of the remaining accessions. In most of these accessions, fruit were oblate with white flesh. 
Table 6. Mean values (of three replications of eight fruit each), standard deviation of the mean, and range of four biochemical traits of melon fruit flesh of 40 Brazilian melon landraces collected from nine states in northeastern and three commercial hybrids.

\begin{tabular}{|c|c|c|c|c|c|c|c|c|}
\hline \multirow[b]{2}{*}{ Accession } & \multicolumn{2}{|c|}{$\mathrm{TSS}^{\mathrm{z}}$} & \multicolumn{2}{|c|}{$\mathrm{pH}^{\mathrm{y}}$} & \multicolumn{2}{|c|}{$\mathrm{AAS}^{\mathrm{x}}$} & \multicolumn{2}{|c|}{$\mathrm{TA}^{\mathrm{w}}$} \\
\hline & Mean \pm SD & Range & Mean \pm SD & Range & Mean \pm SD & Range & Mean \pm SD & Range \\
\hline MA-1 & $3.3 \pm 0.9$ & $2.5-3.5$ & $4.4 \pm 0.3$ & $4.0-4.5$ & $3.99 \pm 0.1$ & $3.10-4.19$ & $0.11 \pm 0.05$ & $0.10-0.13$ \\
\hline MA-2 & $5.4 \pm 0.3$ & $4.1-5.5$ & $6.1 \pm 0.3$ & $5.8-6.2$ & $4.81 \pm 2.4$ & $4.10-5.69$ & $0.09 \pm 0.04$ & $0.05-0.12$ \\
\hline MA-4 & $4.9 \pm 1.2$ & $4.1-5.0$ & $4.7 \pm 0.1$ & $4.6-4.9$ & $9.11 \pm 0.4$ & $8.78-9.16$ & $0.18 \pm 0.03$ & $0.10-0.19$ \\
\hline MA-5 & $4.0 \pm 0.2$ & $3.7-4.2$ & $5.9 \pm 0.4$ & $5.7-6.0$ & $4.71 \pm 0.5$ & $4.00-4.91$ & $0.05 \pm 0.03$ & $0.03-0.09$ \\
\hline MA-6 & $6.1 \pm 0.6$ & $5.6-6.3$ & $5.6 \pm 0.2$ & $5.4-5.7$ & $4.87 \pm 0.4$ & $4.20-5.12$ & $0.20 \pm 0.09$ & $0.19-0.33$ \\
\hline PI-2 & $5.0 \pm 0.3$ & $4.6-5.5$ & $5.5 \pm 0.3$ & $5.0-5.7$ & $5.07 \pm 0.1$ & $4.89-5.54$ & $0.11 \pm 0.02$ & $0.08-0.12$ \\
\hline PI-3 & $5.0 \pm 1.7$ & $4.3-5.3$ & $4.6 \pm 1.2$ & $4.0-4.6$ & $5.12 \pm 0.2$ & $5.00-6.19$ & $0.13 \pm 0.09$ & $0.10-0.14$ \\
\hline CE-1 & $4.2 \pm 0.6$ & $4.0-5.0$ & $5.9 \pm 0.6$ & $5.5-6.0$ & $6.63 \pm 0.2$ & $5.78-6.50$ & $0.12 \pm 0.09$ & $0.11-0.13$ \\
\hline CE-2 & $3.8 \pm 0.6$ & $3.1-4.5$ & $6.0 \pm 0.3$ & $5.7-6.1$ & $4.81 \pm 0.1$ & $4.20-4.98$ & $0.05 \pm 0.03$ & $0.04-0.07$ \\
\hline CE-3 & $4.2 \pm 0.9$ & $3.6-4.3$ & $4.8 \pm 0.5$ & $4.5-4.9$ & $4.35 \pm 0.2$ & $4.00-4.89$ & $0.27 \pm 0.04$ & $0.23-0.29$ \\
\hline CE-4 & $4.3 \pm 0.2$ & $3.7-4.4$ & $3.0 \pm 0.3$ & $2.9-3.2$ & $3.88 \pm 0.3$ & $3.20-3.89$ & $0.59 \pm 0.01$ & $0.51-0.59$ \\
\hline $\mathrm{RN}-5$ & $4.2 \pm 1.1$ & $3.0-4.9$ & $4.6 \pm 0.1$ & $4.3-4.8$ & $6.31 \pm 0.3$ & $5.64-6.40$ & $0.13 \pm 0.03$ & $0.10-0.16$ \\
\hline RN-6 & $4.4 \pm 0.3$ & $3.6-4.6$ & $6.0 \pm 0.4$ & $5.7-6.1$ & $4.66 \pm 0.1$ & $4.10-4.78$ & $0.03 \pm 0.05$ & $0.02-0.06$ \\
\hline $\mathrm{RN}-7$ & $6.2 \pm 0.2$ & $5.1-6.3$ & $5.7 \pm 0.4$ & $5.5-5.9$ & $4.81 \pm 0.1$ & $4.33-5.10$ & $0.16 \pm 0.11$ & $0.13-0.18$ \\
\hline $\mathrm{RN}-8$ & $5.2 \pm 0.2$ & $4.9-5.5$ & $4.7 \pm 0.3$ & $4.3-6.0$ & $5.38 \pm 0.1$ & $5.21-5.97$ & $0.16 \pm 0.01$ & $0.15-0.18$ \\
\hline RN-9 & $4.3 \pm 0.2$ & $3.0-4.4$ & $5.0 \pm 0.8$ & $4.4-5.1$ & $5.54 \pm 0.1$ & $5.15-5.89$ & $0.11 \pm 0.03$ & $0.09-0.12$ \\
\hline PB-1 & $6.5 \pm 0.9$ & $6.0-6.6$ & $6.0 \pm 0.2$ & $5.8-6.1$ & $4.24 \pm 0.3$ & $3.96-4.44$ & $0.05 \pm 0.08$ & $0.04-0.07$ \\
\hline PB-2 & $3.6 \pm 0.2$ & $3.2-3.7$ & $5.5 \pm 0.4$ & $5.2-5.6$ & $5.07 \pm 0.3$ & $4.76-5.12$ & $0.10 \pm 0.09$ & $0.06-0.11$ \\
\hline PB-3 & $6.6 \pm 1.1$ & $6.1-6.7$ & $5.6 \pm 0.5$ & $5.2-5.6$ & $4.3 \pm 0.3$ & $4.00-4.89$ & $0.11 \pm 0.04$ & $0.08-0.12$ \\
\hline PB-4 & $5.4 \pm 0.7$ & $5.0-5.5$ & $5.5 \pm 0.7$ & $5.2-5.7$ & $4.04 \pm 0.1$ & $3.95-4.78$ & $0.11 \pm 0.01$ & $0.10-0.14$ \\
\hline PE-1 & $6.4 \pm 0.7$ & $5.7-6.5$ & $5.5 \pm 0.4$ & $5.3-5.6$ & $4.50 \pm 0.2$ & $3.92-4.60$ & $0.14 \pm 0.06$ & $0.11-0.17$ \\
\hline PE-2 & $6.5 \pm 0.7$ & $6.0-6.8$ & $5.5 \pm 0.2$ & $5.4-5.8$ & $5.18 \pm 0.4$ & $4.10-5.76$ & $0.13 \pm 0.09$ & $0.12-0.15$ \\
\hline BA-1 & $4.0 \pm 0.7$ & $3.1-4.5$ & $4.5 \pm 0.6$ & $4.0-4.7$ & $6.11 \pm 0.2$ & $5.31-6.34$ & $0.21 \pm 0.11$ & $0.19-0.23$ \\
\hline BA-2 & $6.7 \pm 0.5$ & $5.1-6.8$ & $5.9 \pm 0.3$ & $5.6-6.1$ & $4.45 \pm 0.3$ & $3.98-5.12$ & $0.16 \pm 0.06$ & $0.13-0.18$ \\
\hline BA-3 & $3.9 \pm 0.7$ & $2.8-4.6$ & $4.9 \pm 0.9$ & $4.6-5.1$ & $5.44 \pm 0.4$ & $5.10-5.69$ & $0.12 \pm 0.90$ & $0.09-0.14$ \\
\hline BA-4 & $3.1 \pm 0.3$ & $3.0-3.8$ & $4.9 \pm 0.7$ & $4.7-5.0$ & $5.07 \pm 0.1$ & $4.45-5.63$ & $0.16 \pm 0.11$ & $0.14-0.17$ \\
\hline BA-5 & $3.2 \pm 0.2$ & $2.9-3.7$ & $4.9 \pm 0.3$ & $4.6-5.1$ & $5.12 \pm 0.6$ & $4.78-5.33$ & $0.09 \pm 0.09$ & $0.06-0.11$ \\
\hline In-01 & $9.0 \pm 0.9$ & $5.8-10.4$ & $6.1 \pm 0.5$ & $5.8-6.2$ & $5.02 \pm 0.1$ & $4.87-5.63$ & $0.11 \pm 0.05$ & $0.09-0.13$ \\
\hline In-02 & $9.2 \pm 0.4$ & $8.9-11.1$ & $5.9 \pm 0.9$ & $5.6-6.0$ & $5.85 \pm 0.6$ & $4.56-6.10$ & $0.13 \pm 0.08$ & $0.10-0.15$ \\
\hline In-03 & $9.0 \pm 0.5$ & $8.8-10.7$ & $6.0 \pm 0.1$ & $5.8-6.1$ & $4.5 \pm 0.2$ & $3.90-4.60$ & $0.07 \pm 0.02$ & $0.05-0.10$ \\
\hline$F^{\mathrm{v}}$ & 9.5 & & 3.6 & & 2.05 & & 2.18 & \\
\hline SE & 0.5 & & 0.4 & & 0.06 & & 0.11 & \\
\hline $\mathrm{LSD}^{\mathrm{u}}$ & 0.8 & & 0.6 & & 0.12 & & 0.20 & \\
\hline
\end{tabular}

zTotal soluble solids (\%); measured with a digital refractometer.

${ }^{\mathrm{y}} \mathrm{pH}$ measured with a $\mathrm{pH}$ meter.

${ }^{\mathrm{x}}$ Titratable acidity (\%); quantified through titration of a fruit juice sample with $0.05 \mathrm{~N} \mathrm{NaOH}$.

${ }^{w}$ Ascorbic acid (mg/100 $\mathrm{g}$ of fruit flesh); measured according to the process described by Dhillon et al. (2009).

${ }^{\text {v }} F=$ estimated by Snedecor's $F$ test.

"Least significant difference by $t$ test.

The other accessions were further separated into two groups (groups II and III in Fig. 3B) according to the second principal component, which accounted for the $23.83 \%$ of the total variation. Accessions of these two groups shared characteristics of earlier flowering $(26.23 \pm 0.47$ and $26.92 \pm 0.67$ DAT, respectively) and larger fruit $(1.816 \pm 0.17$ and $1.726 \pm 0.15 \mathrm{~kg}$, respectively), although accessions in group III had smaller seeds $(2.58 \pm 0.16 \mathrm{~g})$ than those in group II $(3.80 \pm 0.18 \mathrm{~g})$. 

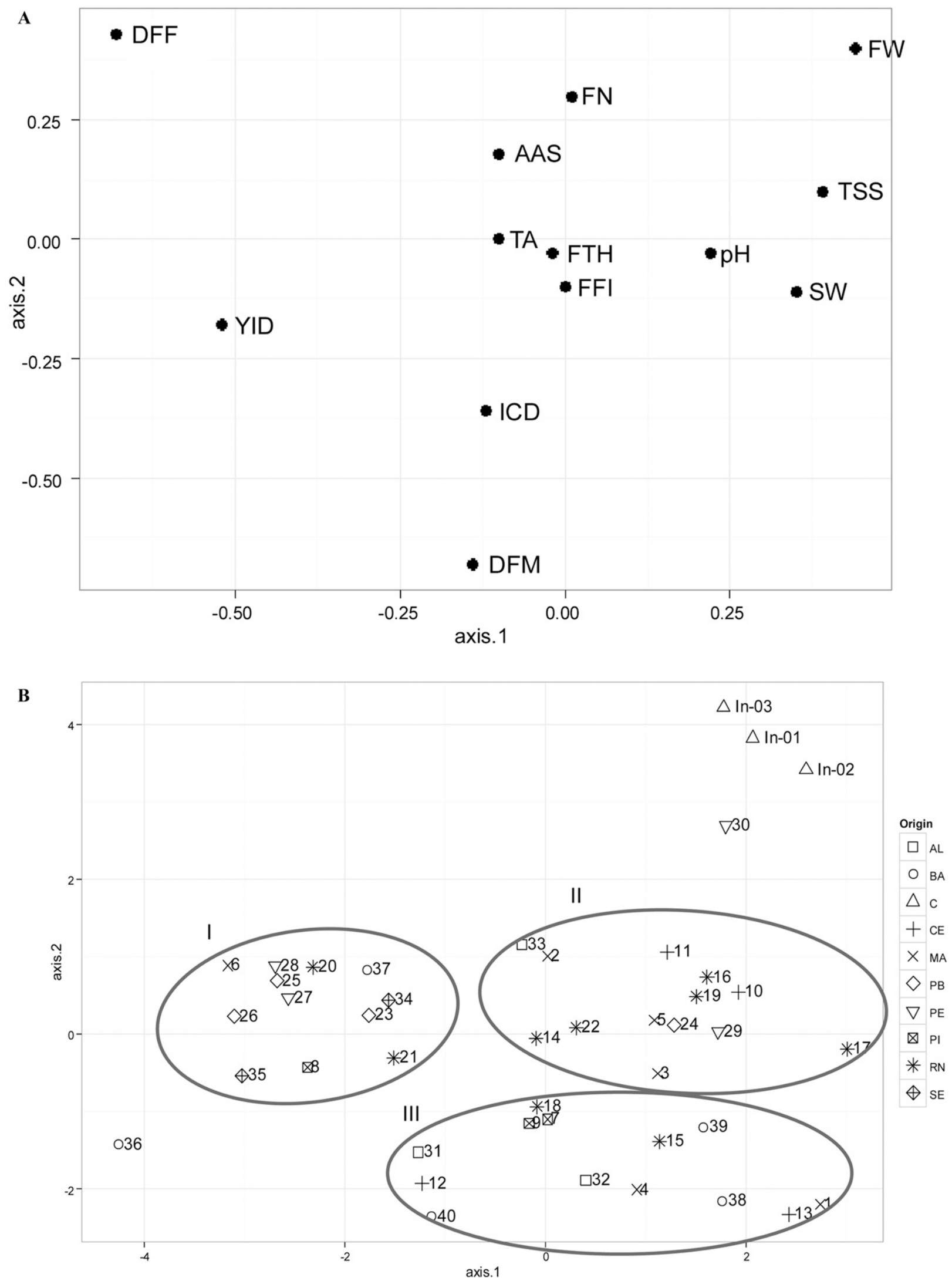

Fig. 3. Principal component analysis of quantitative flower, fruit, and seed traits of 40 melon landraces collected in northeastern Brazil and three control hybrids showing (A) the relationship and contribution to the principal components 1 and 2 (axes 1 and 2) of the different traits assessed in the morphological characterization (trait names according to Tables 4-6) and (B) the relationships among these genotypes using these quantitative characters. The symbols in the legend indicate the respective states from which the landraces were collected: Alagoas (AL), Bahia (BA), references (C), Ceará (CE), Maranhão (MA), Paraíba (PB), Pernambuco (PE), Piauí (PI), Rio Grande do Norte (RN), and Sergipe (SE). Accession numbers are those from Table 1. 
Group III accessions had higher yields $\left(34.81 \pm 2.44 \mathrm{t} \cdot \mathrm{ha}^{-1}\right)$ than those of group II $\left(22.12 \pm 2.10 \mathrm{t} \cdot \mathrm{ha}^{-1}\right)$ and firmer fruit, lower TSS, and more acidic flesh $\left(16.7 \pm 0.86\right.$ vs. $19.82 \pm 1.11 \mathrm{~N} \cdot \mathrm{m}^{-2}$, $4.53 \% \pm 0.18 \%$ vs. $3.96 \% \pm 0.20 \%$, and pH $5.80 \pm 0.9$ vs. $4.81 \pm$ 0.25 , in groups II and III, respectively), with some accessions exhibiting the highest levels of titratable acid and ascorbic acid contents (Table 6). Accessions of group II were quite variable in fruit shape and flesh color, whereas those of group III were mostly elongated with white flesh. Within each of these three groups, there was much variability with regard to the analyzed traits, irrespective of the site of collection.

The accession BA-1 was separated from the other three accessions collected in Bahia. It bears distinctive characteristics (nonclimacteric and quite prolific, with small seeds and fruit), although it shares the characteristics of low sugar content, acidic flesh and monoecious sex type with the other Brazilian germplasms (Tables 3-6). Only one Brazilian accession (PE-4, from Pernambuco) was grouped with the control Amarillo hybrids; it was the only accession with TSS $>7.0 \%$ among the Brazilian germplasms (Table 6).

Molecular Characterization USING SSR loci. All SSR markers were polymorphic with a total of 64 alleles across the full set of melon accessions, with a mean of 4.9 alleles per locus within a range interval of 3 (CMMS 15-4) to 8 (CMMS 35-4) (Table 7). A detailed table including the estimated fragment sizes amplified at each locus in each landrace has been included as supplementary material (Supplemental Table 2).

The major allele frequency had a mean of 0.54 , with values ranging from 0.35 (CMMS 35-4) to 0.72 (CMMS 30-3). The mean PIC value, an indicator of loci polymorphism, was 0.55 and ranged from 0.42 (CMMS 2-3) to 0.74 (CMMS 35-4). The observed heterozygosity was 0.36 within a range interval of 0.08 (CMMS 30-3) to 0.85 (CMMS 33-2).

The genetic variability of the Brazilian landraces was compared with that of the reference accessions (Table 7). Despite the reference accessions included a representation of

Table 7. Genetic variability detected with simple sequence repeat markers in 40 Brazilian melon landraces from nine states of northeastern and 21 references calculated with Powermarker 3.25 (Liu and Muse, 2005).

\begin{tabular}{lcccccc}
\hline Markers $^{\mathrm{z}}$ & Alleles (no.) & Genotypes (no.) & MAF $^{\mathrm{y}}$ & Gene diversity & Het $^{\mathrm{x}}$ & PIC $^{\mathrm{w}}$ \\
\hline CMMS 2-3 & 4 & 5 & 0.67 & 0.48 & 0.20 & 0.42 \\
CMMS 4-3 & 6 & 9 & 0.64 & 0.56 & 0.19 & 0.53 \\
CMMS 12-6 & 6 & 10 & 0.47 & 0.66 & 0.36 & 0.61 \\
CMMS 15-4 & 3 & 5 & 0.70 & 0.46 & 0.09 & 0.42 \\
CMMS 22-2 & 4 & 8 & 0.39 & 0.70 & 0.70 & 0.65 \\
CMMS 24-3 & 4 & 8 & 0.55 & 0.60 & 0.20 & 0.54 \\
CMMS 30-3 & 6 & 7 & 0.72 & 0.46 & 0.08 & 0.44 \\
CMMS 33-1 & 6 & 11 & 0.55 & 0.63 & 0.49 & 0.59 \\
CMMS 33-2 & 4 & 5 & 0.54 & 0.60 & 0.85 & 0.54 \\
CMMS 34-8 & 4 & 8 & 0.53 & 0.60 & 0.22 & 0.53 \\
CMMS 34-10 & 4 & 7 & 0.58 & 0.55 & 0.20 & 0.48 \\
CMMS 35-4 & 8 & 12 & 0.35 & 0.77 & 0.59 & 0.74 \\
CMMS 35-5 & 5 & 9 & 0.38 & 0.70 & 0.47 & 0.65 \\
Avg & 4.91 & 8.00 & 0.54 & 0.60 & 0.36 & 0.55 \\
Avg (Brazil) & 3.92 & 5.69 & 0.63 & 0.49 & 0.30 & 0.43 \\
Avg (references) & 4.31 & 5.38 & 0.46 & 0.65 & 0.46 & 0.59 \\
\hline
\end{tabular}

${ }^{\mathrm{z}}$ Details about the used simple sequence repeats are in Chiba et al. (2003).

${ }^{\mathrm{y}}$ Major allele frequency.

${ }^{\mathrm{x}}$ Observed heterozygosity.

wPolymorphism information content. most of the horticultural groups of the species, differences in the mean allele number and in the mean genetic diversity were not high between the reference and Brazilian accessions (4.31 vs. 3.92 alleles per locus and 0.65 vs. 0.49 gene diversity, respectively). Similar trends were observed in the PIC and heterozygosity values.

The molecular relationships between the Brazilian landraces and the references, estimated using FCA, are shown in Fig. 4A. According to the first factor, Brazilian accessions were grouped apart from the references, suggesting a different genetic diversity in this material. In general, they were more similar to the reference accessions of ssp. agrestis (Momordica, Makuwa, Chinensis, Acidulus, Tibish and wild agrestis) and to the intermediate groups (Dudaim and Flexuosus) but far apart from the Inodorus, Cantalupensis, Reticulatus, and Ameri accessions (ssp. melo) included herein.

For a better view of the relationships of Brazilian accessions with the references, only the closest accessions of ssp. agrestis were plotted in Fig. 4B. The accession BA-1, which was clearly different in the morphological analysis, was also molecularly separated from the remaining accessions. In fact, BA-1 (nonclimacteric, and with small seeds and fruit) was genetically closer to the African Acidulus reference.

The dispersion of Brazilian accessions along the second factor is shown in Fig. 4B. The group of accessions in the upper part of the graph shows a clear molecular similarity with Momordica references from India. These were mainly accessions of the morphological groups II and III, which were quite variable in their morphological traits. In the lower part of the graph, grouped Brazilian accessions more similar to the Far Eastern references (Chinensis and Makuwa) are shown. These accessions were mostly grouped (group I) according to the morphological analyses (Fig. 3B). Most of the remaining Brazilian accessions were intermediate between this molecular group and those similar to Momordica, as were the Asian Flexuosus and Dudaim references. Similar to the morphological results, molecular grouping of accessions according to the site of collection was not observed.

\section{Discussion}

After its domestication, melon was dispersed throughout Asia and Africa (Kerje and Grum, 2000), and through the centuries it has undergone an intense process of diversification, becoming the most polymorphic species of the genus Cucumis (Kirkbride, 1993).

Today, melon shows great variation in morphological, biochemical, and physiological traits (Burger et al., 2010; Esteras et al., 2013; Leida et al., 2015). Much effort has been made to quantify and characterize the variation observed in melons from several primary and secondary centers of diversity such as India (Dhillon et al., 2012; Malik et al., 2014; Roy et al., 2012), Africa (Mliki et al., 2001; Mohamed and 


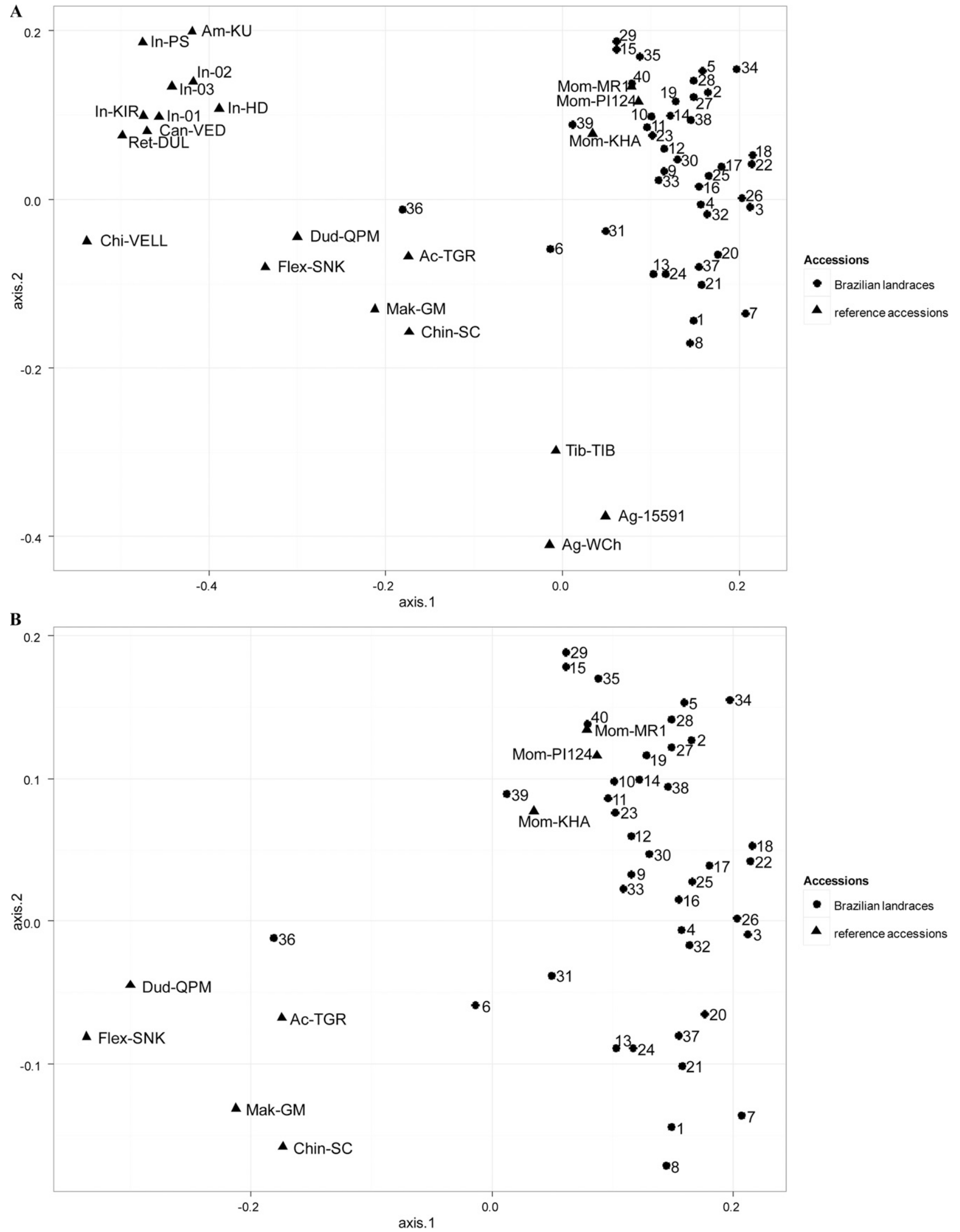

Fig. 4. Factor correspondence analyses (FCA) of 13 simple sequence repeat loci to determine the genetic relationships of 40 melon landraces collected in northeastern Brazil and 21 representatives of 13 horticultural groups of Cucumis melo ssp. agrestis and ssp. melo. (A) FCA with the 40 landraces and 21 representative accessions and (B) FCA with the 40 landraces and eight ssp. agrestis accessions. Numbers of the landraces are given in Table 1. Codes of representative accessions are given in Table 2. Brazilian melon landraces $(\bullet)$ and representative accessions $(\boldsymbol{\Delta})$ from diverse origins. 
Yousif, 2004), China (Luan et al., 2008), Greece (Staub et al., 2004), Turkey (Frary et al., 2013), Tunisia (Trimech et al., 2013), Korea (Park et al., 2013), Iran (Raghami et al., 2014), and Spain (Escribano et al., 2012; Esteras et al., 2013), among others.

Northeastern Brazil has been referred to as a secondary center of diversity for watermelon (Romão, 2000). Other cucurbits, such as gherkin and bitter gourd (Momordica charantia), introduced by African slaves, also spread out across the entire northeastern region (Queiróz, 2004). Although Brazil has not been previously reported as a diversification center for melons, this study shows that in northeastern Brazil, a large variability of this species currently exists, maintained in the form of landraces. These landraces are morphologically variable for most of the traits analyzed, such as flowering time, seed weight, fruit shape and weight, flesh color and firmness, number of fruit per vine, and yield. The genetic basis of some of these traits has been previously studied, as most of them are controlled by diverse genomic regions. In fact, several quantitative trait loci involved in their variation have been reported (Diaz et al., 2011; Fernández-Trujillo et al., 2011).

However, this morphological variability seems to be lower than that reported in germplasm from the area of origin and primary diversification such as India, China, Turkey, or Iran (Dhillon et al., 2007, 2009; Frary et al., 2013; Luan et al., 2008; Raghami et al., 2014). In fact, most of the Brazilian landraces share some features such as monoecious sex type and climacteric ripening behavior. They also develop acidic fruit, usually elongated to oblate, with a low to medium sugar content, characteristics that fit in more with those of Asian melons belonging to the Momordica (snapmelon), Flexuosus and Chate (snake and cucumber melon), and Far Eastern (Makuwa and Chinensis) groups (Leida et al., 2015; Pitrat, 2008; Stepansky et al., 1999).

The morphological analysis revealed three groups. The features of group I include smaller seeds and fruit, quite uniform oblate to elongated fruit shape and white flesh with the highest TSS values among the Brazilian landraces, which fit more with the Far Eastern Makuwa melons, although they are usually andromonoecious.

The characteristics of group III, with earlier flowering accessions, larger seeds and fruit, elongated fruit with less sweet and more acidic flesh, fit better with traits of the Momordica and/or the Flexuosus and Chate groups. Acidic and low-sugar flesh is also typical of the Acidulus group, but this is usually nonclimacteric. However, because we have found one accession (BA-1, the only nonclimacteric), which fits better with the Acidulus group, some of these acidic accessions could be intermediate types derived from intergroup crosses. This is not unexpected as no intergroup crossing barriers have been previously reported.

Group II was more variable in fruit shape and flesh color, and included the accessions with the largest seeds and, in general, with the softest flesh. These traits also fit with the variability reported in the Momordica group, but some characteristically elongated ribbed fruit resembles that of the Flexuosus and Chate groups (with larger seeds and fruit) traditionally classified in ssp. melo.

Because variation in morphological traits was still high within these three groups, the molecular results provided complementary information to improve the classification and detect mixtures. Our molecular results also support a high molecular diversity, only slightly lower than that of the reference controls, which represent most of the sub-specific horticultural groups, and higher than that reported in Brazilian germplasms analyzed in previous studies. In fact, the mean PIC that we observed in the Brazilian collection $(0.43)$ is greater than that estimated by Aragão et al. [2013 (PIC = 0.32)] in the germplasm of only some of the northeastern Brazilian states sampled in our study. However, our PIC value is lower than the estimates obtained by other authors who worked with Indian collections [ranging from 0.54 to 0.70 (Dhillon et al., 2007, 2009; Fergany et al., 2011; Malik et al., 2014; Roy et al., 2012)].

The molecular results also confirm the genetic relationships of the Brazilian accessions with germplasms from the centers of origin and primary diversification. They were quite different from reference accessions belonging to the three main commercial groups, Inodorus, Cantalupensis, and Reticulatus, and could be undoubtedly classified together with accessions mostly of ssp. agrestis. Within ssp. agrestis, some reference accessions from India of the Momordica group were genetically closer to a group of Brazilian accessions from different sampling sites, including accessions from all Brazilian states. This result is consistent with the morphological characterization, as most of these accessions belonged to the morphological groups II and III, which showed morphological characteristics close to the Momordica group. In addition, most of these Brazilian landraces have additional characteristic features of snapmelons, including fruit cracking and mealy flesh texture. Previous morphological evaluations of Brazilian germplasms from some northeastern (Aragão et al., 2013) and southern (Neitzke et al., 2009) states described fruit with similar characteristics.

The Momordica germplasm from India has been proven to have a quite large genetic diversity (Dhillon et al., 2007, 2009). Some authors have proposed that India has a central position in melon origin and is where Oriental and Occidental melons developed (Blanca et al., 2012). Fruit of the Momordica group were intensively cultivated in northern India in the past and are currently known in almost all parts of the country. Immature fruit are eaten raw, cooked, or pickled (Dhillon et al., 2007). The cultivation of Momordica outside of India is not common, although it has been reported in other Asian countries (Fujishita, 2004). Fruit of the Brazilian landraces similar to Momordica are grown in northeastern Brazil by local farmers, not only for selfconsumption but also for marketing. When ripe, the fruit are consumed in desserts with sugar; they are also used for the production of juices and sweets. Moreover, this type of fruit is also commercially produced in southern Brazil, where it is known as "snow melon," "snowball," or "snow cucumber"; some types of local Momordica melons are also called "melon caipira." Because Momordica melons are mostly found in India (Dhillon et al., 2009), it is remarkable that their farming tradition and variability of the landraces is found in different Brazilian regions. It is not known how or when the introduction of these snapmelons occurred in Brazil. Our initial hypothesis was that these landraces were introduced by European immigrants, because African slaves probably did not cultivate this type of melon on the African continent.

In addition, consistent with the morphological analysis, another large group of landraces from different Brazilian states, mostly belonging to the morphological group I, seems to be genetically closer to the Chinensis and Makuwa references, which are cultivated in Asian countries but are considered to be exotic in Occidental markets. The molecular analysis also 
confirmed the intermediate position of some melons of the morphological group II, which resembles Chate melons. Chate melons and, in general, the Flexuosus group are considered to be the oldest form of cultivated melons, which are still appreciated in southern Europe, north of Africa, and central Asia (McCreight et al., 2013). The remaining Brazilian landraces seem to be molecularly intermediate between the Momordica and the Far Eastern melons and might represent intergroup crossings, as no sexual barriers separate any of these groups.

Our results show that the Brazilian germplasm is mostly composed of Momordica/Flexuosus-Chate/Makuwa-Chinensis melons as well as other less common groups, such as the nonclimacteric BA-1, which was morphologically and molecularly more similar to Acidulus. The Acidulus group is native of the southern India humid tropics. The fruit of this group is eaten in typical Indian salads and dishes such as the "sambhar," a vegetable stew with tamarind [Tamarindus indica (Fergany et al., 2011)]. In Brazil, the fruit of this type of nonclimacteric landraces is eaten in salads when immature, but, as shown in this study, this is not a common type of melon among Brazilian landraces.

Taken together, morphological and molecular results show that the Brazilian landraces are largely diverse. The fact that these landraces were not grouped according to the sites of sampling suggests that small producers have promoted genetic material exchange. A more comprehensive survey of both northeastern Brazil and worldwide reference germplasms would be necessary for a better definition of the Brazilian material origin and diversity.

The analysis of the extant diversity, both morphological and molecular, in local germplasms, may be of utility for germplasm curators in providing relevant information for the preservation of the genetic variability associated with traditional Brazilian agriculture. In fact, genetic erosion of Brazilian landraces is starting to occur, mainly in the states of Rio Grande do Norte and Ceará, which are the main Brazilian producers and exporters of melon. These states are adopting intensive agricultural systems with high investments in modern irrigation techniques, high inputs, and hybrid seeds. Small producers in these states are replacing the traditional landraces with more profitable hybrids.

The characterization of this Brazilian material is also interesting from a breeding point of view. The germplasm from Asia, mainly of Indian origin, is the source of most of the alleles that have been used in melon breeding programs for resistance to pests and diseases (Dhillon et al., 2012). The most prominent case is the use of Indian germplasms in breeding programs for resistance to the fungi Podosphaera xanthii and Golovinomyces cichoracearum, the main causal agents of powdery mildew in cucurbits. A preliminary study of the extant Brazilian germplasm identified sources of resistance to races 1, 2, and 5, which are found in melon production fields throughout northeastern Brazil (data not shown). This confirms that the Brazilian germplasm is potentially useful for the improvement of the melon market.

\section{Literature Cited}

Aragão, F.A.S., J. Torres Filho, G.H.S. Nunes, M.A. Queiróz, P.N. Bordallo, G.S.C. Buso, M.A. Ferreira, Z.P. Costa, and F. Bezerra Neto. 2013. Genetic divergence among accessions of melon from traditional agriculture of the Brazilian northeast. Genet. Mol. Res. 12:6356-6371.
Blanca, J.M., C. Esteras, P.Z. Areitioaurtena, D. Pérez, V. FernandezPedrosa, C. Collado, R.R. de Pablos, A. Ballester, M.C. RoigMontaner, J. Cañizares, and M.B. Picó. 2012. Transcriptome sequencing for SNP discovery across Cucumis melo. BMC Genomics 13:280.

Burger, Y., H.S. Paris, R. Cohen, N. Katzir, Y. Tadmor, and E. Lewinsohn. 2010. Genetic diversity of Cucumis melo. Hort. Rev. 36:165-198.

Chiba, N., K. Suwabe, T. Nunome, and M. Hirai. 2003. Development of microsatellite markers in melon (Cucumis melo L.) and their application to major cucurbit crops. Breed. Sci. 53:21-27.

Delwing, A.B., L.B. Franke, and I.B.I. Barros. 2007. Qualidade de sementes de acessos de melão crioulo (Cucumis melo L.). Revista Brasileira de Sementes 29:187-194.

Dhillon, N.P.S., A.J. Monforte, M. Pitrat, S. Pandey, P.K. Singh, K.R. Reitsma, J. Garcia-Mas, A. Sharma, and J.D. McCreight. 2012. Melon landraces of India: Contributions and importance. Plant Breed. Rev. 35:85-150.

Dhillon, N.P.S., J. Singh, M. Fergany, A.J. Monforte, and A.K. Sureja. 2009. Phenotypic and molecular diversity among landraces of snapmelon (Cucumis melo var. momordica) adapted to the hot and humid tropics of eastern India. Plant Genet. Resources 7:291300.

Dhillon, N.P.S., R. Ranjana, K. Singh, I. Eduardo, A.J. Monforte, M. Pitrat, N.L. Dhillon, and P.P. Singh. 2007. Diversity among landraces of indian snapmelon (Cucumis melo var. momordica). Genet. Resources Crop Evol. 54:1267-1283.

Diaz, A., M. Fergany, G. Formisano, P. Ziarsolo, J. Blanca, Z. Fei, J.E. Staub, J.E. Zalapa, H.E. Cuevas, G. Dace, M. Oliver, N. Boissot, C. Dogimont, M. Pitrat, M.R. Hofstede, P. Koert, R. Harel-Beja, G. Tzuri, V. Portnoy, S. Cohen, A. Schaffer, N. Katzir, Y. Xu, H. Zhang, N. Fukino, S. Matsumoto, J. Garcia-Mas, and A.J. Monforte. 2011. A consensus linkage map for molecular markers and quantitative trait loci associated with economically important traits in melon (Cucumis melo L.). BMC Plant Biol. 11:1471-2229.

Doyle, I.J. and J.L. Doyle. 1990. Isolation of plant from fresh tissue. Focus 12:13-15.

Escribano, S., A. Lázaro, H.E. Cuevas, A.I. López-Sesé, and J.E. Staub. 2012. Spanish melons (Cucumis melo L.) of the Madrid provenance: A unique germplasm reservoir. Genet. Resources Crop Evol. 59:359-373.

Esteras, C., F. Nuez, and M.B. Picó-Sirvent. 2012. Genetic diversity studies in cucurbits using molecular tools, p. 225-255. In: Y.H. Wang, T.K. Behera, and C. Kole (eds.). Genetics, genomics and breeding of crop plants: Genetics, genomics and breeding of cucurbits. Science Publ., Enfield, NH.

Esteras, C., G. Formisano, C. Roig, A. Díaz, J. Blanca, J. Garcia-Mas, M.L. Gómez-Guillamón, A.I. Lopéz-Sesé, A. Lázaro, A.J. Monforte, and B. Picó. 2013. SNP genotyping in melons: Genetic variation, population structure, and linkage disequilibrium. Theor. Appl. Genet. 126:1285-1303.

Fergany, M., B. Kaur, A.J. Monforte, M. Pitrat, C. Rys, H. Lecoq, N.P.S. Dhillon, and S.S. Dhaliwal. 2011. Variation in melon (Cucumis melo) landrances adapted to the humid tropics of sourthern India. Genet. Resources Crop Evol. 58:225-243.

Fernández-Trujillo, J.P., B. Picó, J. Garcia-Mas, J.M. Álvarez, and A.J. Monforte. 2011. Breeding for fruit quality in melon, p. 261278. In: M.A. Jenks and P.J. Bebeli (eds.). Breeding for fruit quality. Wiley, Hoboken, NJ.

Food and Agriculture Organization of the United Nations (FAO). 2014. FAOSTAT, ProdSTAT-Crops \#1. 10 Oct. 2014. <http:// faostat.fao.org/>.

Frary, A., H.O. Sigva, A. Tan, T. Taskın, A. Inal, S. Mutlu, M. Haytaoglu, and S. Doganlar. 2013. Molecular genetic diversity in the Turkish national melon collection and selection of a preliminary core set. J. Amer. Soc. Hort. Sci. 138:50-56.

Fujishita, N. 2004. Pedigree of melon (Cucumis melo) in ancient Japan. Intl. Symp. Asian Plant Diversity and Systematics, Sakura, Japan, 29 July-1 Aug. 2004 (Abstr. OS-3). p. 2. 
Garcia-Mas, G., A. Benjak, W. Sanseverino, M. Bourgeois, G. Mir, V.M. González, E. Hénaff, F. Câmara, L. Cozzuto, E. Lowy, T. Alioto, S. Capella-Gutiérrez, J. Blanca, J. Cañizares, P. Ziarsolo, D. GonzalezIbeas, L. Rodríguez-Moreno, M. Droeg, L. Duh, M. Alvarez-Tejado, B. Lorente-Galdos, M. Meléc, L. Yang, Y. Weng, A. Navarro, T. Marques-Bonet, M.A. Aranda, F. Nuez, B. Picó, T. Gabaldón, G. Roma, R. Guigóc, J.M. Casacuberta, P. Arús, and P. Puigdomènech. 2012. The genome of melon (Cucumis melo L.). Proc. Natl. Acad. Sci. USA 109:11872-11877.

International Plant Genetic Resources Institute. 2003. Descriptors for melon (Cucumis melo L.). IPGRI, Rome, Italy.

Jeffrey, C. 1980. A review of the Cucurbitaceae. Bot. J. Linn. Soc. 81:233-247.

John, K.J., S. Scariah, V.A.M. Nissar, M. Latha, S. Gopalakrishnan, S.R. Yadav, and K.V. Bhat. 2012. On the occurrece, distribution, taxonomy and genepool relationship of Cucumis callosus (Rottler) Cogn., the wild progenitor of Cucumis melo L. from India. Genet. Resources Crop Evol. 59:1-10.

Kerje, T. and M. Grum. 2000. The origin of melon, Cucumis melo: A review of the literature. Acta Hort. 510:37-44.

Kirkbride, J.H. 1993. Biosystematic monograph of the genus Cucumis (Cucurbitaceae). Parkway Publ., Boone, NC.

Leida, C., C. Moser, C. Esteras, R. Sulpice, J. Lunn, F. De Langen, A.J. Monforte, and B. Picó. 2015. Variability of candidate genes, genetic structure and association with sugar accumulation and climacteric behavior in a broad germplasm collection of melon (Cucumis melo L.). BMC Genet. 16:1-17.

Liu, K. and S.V. Muse. 2005. Powermaker: Integrated analysis environment for genetic marker data. Bioinformatics 21:2128-2129.

López-Sesé, A.I., J.E. Staub, and M.L. Gómez-Guillamón. 2003. Genetic analysis of Spanish melon (Cucumis melo L.) germplasm using a standardized molecular marker array and reference accessions. Theor. Appl. Genet. 108:41-52.

Luan, F., I. Delannay, and J.E. Staub. 2008. Chinese melon (Cucumis melo L.) diversity analyses provide strategies for germplasm curation, genetic improvement, and evidentiary support of domestication patterns. Euphytica 164:445-446.

Luan, F., Y. Sheng, Y. Wang, and J.E. Staub. 2010. Performance of melon hybrids derived from parents of diverse geographic origins. Euphytica 173:1-16.

Malik, A.A., V.K. Vashisht, K. Singh, A. Sharma, D.K. Singh, H. Singh, A.J. Monforte, J.D. McCreight, and N.P.S. Dhillon. 2014. Diversity among melon (Cucumis melo L.) landraces from the Indo-Gangetic plains of India and their genetic relationship with USA melon cultivars. Genet. Resources Crop Evol. 61:1189-1208.

McCreight, J.D., J.E. Staub, T.C. Wehner, and N.P.S. Dhillon. 2013. Gone global: Familiar and exotic cucurbits have Asian origins. HortScience 48:1078-1089.

Melonomics Project Team. 2014. Genome. 10 Oct. 2014. <https:// melonomics.net/>.

Mliki, A., J.E. Staub, S. Zhangyong, and A. Ghorbel. 2001. Genetic diversity in melon (Cucumis melo L.): An evaluation of African germplasm. Genet. Resources Crop Evol. 48:587-597.

Mohamed, E.T.I. and M.T. Yousif. 2004. Indigenous melons (Cucumis melo L.) in Sudan: A review of their genetic resources and prospects for use as sources of disease and insect resistance. Plant Genet. Resour. Newsl. 138:36-42.

Monforte, A.J., J. Garcia-Mas, and S.P. Arús. 2003. Genetic variability in melon based on microsatellite variation. Plant Breed. 122:153-157.

Munger, H.M. and R.W. Robinson. 1991. Nomenclature of Cucumis melo L. Cucurbit Genet. Coop. Rpt. 14:43-44.
Naudin, C. 1859. Essais d'une monographie des espèces et des variétés du genre Cucumis. Annales des Sciences Naturelles Botanique 11:5-87. Nei, M., F. Tajima, and Y. Tateno. 1983. Accuracy of estimated phylogenetic trees from molecular data. II. Gene frequency data. J. Mol. Evol. 19:153-170.

Neitzke, R.S., R.L. Barbieri, G. Heiden, M.V. Büttow, C.S. Oliveira, L.B. Corrêa, J.E. Schwengber, and F.I.F. Carvalho. 2009. Caracterização morfológica e dissimilaridade genética entre variedades crioulas de melão. Horticultura Brasileira 27:534-538.

Nesom, G.L. 2011. Toward consistency of taxonomic rank in wild/ domesticated Cucurbitaceae. Phytoneuron 13:1-33.

Nunes, G.H.S., H. Santos Jr, L.C. Grangeiro, F. Bezerra Neto, C.T.S. Dias, and M.S.M. Dantas. 2011. Phenotypic stability of hybrids of Galia melon in Rio Grande do Norte state, Brazil. An. Acad. Bras. Cienc. 83:1421-1434.

Nunes, G.H.S., J.J. Santos Júnior, F.V. Andrade, F. Bezerra Neto, J.B. Menezes, and E.W.L. Pereira. 2005. Desempenho de híbridos do grupo inodorus em Mossoró. Horticultura Brasileira 23:90-94.

Park, I., J. Kim, J. Lee, S. Kim, O. Cho, K. Yang, J. Ahn, S. Nahm, and H. Kim. 2013. Development of SSR markers by next-generation sequencing of Korean landraces of chamoe (Cucumis melo var. makuwa). Mol. Biol. Rpt. 40:6855-6862.

Pitrat, M. 2008. Melon (Cucumis melo L.), p. 283-315. In: J. Prohens and F. Nuez (eds.). Handbook of plant breeding-Vegetables. Vol. 1. Springer, New York, NY.

Pitrat, M., P. Hanelt, and K. Hammer. 2000. Some comments on infraspecific classification of cultivars of melon. Acta Hort. 510:29-36.

Queiróz, M.A. 2004. Germplasm of Cucurbitaceae in Brazil. Crop Breeding Appl. Biotechnol. 4:377-383.

Raghami, M., A.I. Loìpez-Sesé, M.R. Hasandokht, Z. Zamani, M.R.F. Moghadam, and A. Kashi. 2014. Genetic diversity among melon accessions from Iran and their relationships with melon germplasm of diverse origins using microsatellite marker. Plant Syst. Evol. 300:139-151.

Robinson, R.W. and D. Decker-Walters. 1997. Cucurbits. CAB International, Wallingford, UK.

Romão, R.L. 2000. Northeast Brazil: A secondary center of diversity for watermelon (Citrullus lanatus). Genet. Resources Crop Evol. 47:207-213.

Roy, A., S.S. Bal, M. Fergany, S. Kaur, H. Singh, A.A. Malik, J. Singh, A.J. Monforte, and N.P.S. Dhillon. 2012. Wild melon diversity in India (Punjab State). Genet. Resources Crop Evol. 59:755-767.

Sebastian, P., H. Schaefer, I.R. Telford, and S.S. Renner. 2010. Cucumber (Cucumis sativus) and melon (C. melo) have numerous wild relatives in Asia and Australia, and the sister species of melon is from Australia. Proc. Natl. Acad. Sci. USA 107:14269-14273.

Staub, J.E., A.I. Lopez-Sesé, and N. Fanourakis. 2004. Diversity among melon landraces (Cucumis melo L.) from Greece and their genetic relationships with other melon germplasm of diverse origins. Euphytica 136:151-166.

Stepansky, A., I. Kovalski, and R. Perl-Treves. 1999. Intraspecific classification of melons (Cucumis melo L.) in view of their phenotypic and molecular variation. Plant Syst. Evol. 217:313-332.

Trimech, R., Y. Zaouali, A. Boulila, L. Chabchoub, I. Ghezal, and M. Boussaid. 2013. Genetic variation in Tunisian melon (Cucumis melo L.) germplasm as assessed by morphological traits. Genet. Resources Crop Evol. 60:1621-1628.

Tzitzikas, E.N., A.J. Monforte, A. Fatihi, Z. Kypriotakis, T.A. Iacovides, I.M. Ioannides, and P. Kalaitzis. 2009. Genetic diversity and population structure of traditional Greek and Cypriot melon cultigens (Cucumis melo L.) based on simple sequence repeat variability. HortScience 44:1820-1824. 
Supplemental Table 1. Primers of the 13 simple sequence repeats (Chiba et al., 2003) used for the analysis of the Brazilian melon landraces and references. Their physical position and scaffold in the last version of the melon genome v3.5 (Melonomics Project Team, 2014) and an estimate of the genetic position, according to the genetic map in melonomics (Garcia-Mas et al., 2012) is included.

\begin{tabular}{|c|c|c|c|c|c|}
\hline SSR marker & Primer sequence & $\begin{array}{l}\text { Expected } \\
\text { amplicon } \\
\text { size (bp) }\end{array}$ & $\begin{array}{l}\text { Physical position } \\
\text { of each primer }\end{array}$ & Scaffold & $\begin{array}{c}\text { Genetic position } \\
\text { in linkage } \\
\text { group }(\mathrm{cM})\end{array}$ \\
\hline \multirow[t]{2}{*}{ CMMS4-3 } & ACCGAAATCATAAGGAACATAAGAG/ & 155 & $2335344-2335320$ & CM3.5_scaffold00026 & $\mathrm{I}(47-50 \mathrm{cM})$ \\
\hline & TATGAGCTGTGTTGTGTATGAAAAC & & $2335189-2335213$ & & \\
\hline \multirow[t]{2}{*}{ CMMS22-2 } & CGTTATACAAGATAGAGATAGAGAG/ & 197 & $917436-917460$ & CM3.5_scaffold00067 & $\mathrm{I}(85-86 \mathrm{cM})$ \\
\hline & TTCAACTAATCCCCAAGACAAACAA & & $917633-917609$ & & \\
\hline \multirow[t]{2}{*}{ CMMS15-4 } & GTCCGCCATCGCCACTACAAATCAA/ & 116 & $2878372-2878396$ & CM3.5_scaffold00011 & IV $(77-79 \mathrm{cM})$ \\
\hline & CTCCGTAAAACCTTCTTCCTCTCTC & & no hits found & & \\
\hline \multirow[t]{2}{*}{ CMMS2-3 } & ATCACCCACCCCACCACTGCCAAAA/ & 251 & $3695074-3695099$ & CM3.5_scaffold00022 & $\mathrm{V}(0 \mathrm{cM})$ \\
\hline & CCTTGAAAAACCACCAACATAACAC & & $3695325-3695301$ & & \\
\hline \multirow[t]{2}{*}{ CMMS24-3 } & GAGGGAGAGAGTTTGTAAAAAAATG/ & 136 & no hits found & CM3.5_scaffold00006 & VI $(0-2 \mathrm{cM})$ \\
\hline & TCGCCAATTACATTACAACCTTTTC & & 87024 & & \\
\hline \multirow[t]{2}{*}{ CMMS34-8 } & TTTCTTACTTTTTGGTTTGGTTCTG/ & 485 & $3954078-3954103$ & CM3.5_scaffold00006 & VI $(29-32 \mathrm{cM})$ \\
\hline & GGCGCTGTGGTGAGTGTCGGGAGAG & & $3954563-3954539$ & & \\
\hline \multirow[t]{2}{*}{ CMMS12-6 } & AACATGATGTGTTTACCAACTTTTT/ & 238 & $2574831-2574807$ & CM3.5_scaffold00038 & VI (49-50cM) \\
\hline & GGTTAAGGGAAAGTGAAGAGATGGT & & $2574593-2574617$ & & \\
\hline \multirow[t]{2}{*}{ CMMS30-3 } & TTCCCACCAGCCCAACGGACACACT/ & 334 & $1164047-1164071$ & CM3.5_scaffold00081 & VII $(30-31 \mathrm{cM})$ \\
\hline & GAGATACAGAAACGACGACTAACCT & & $1164381-1164357$ & & \\
\hline \multirow[t]{2}{*}{ CMMS35-5 } & AACGGGATTTTGGAGGCATATTCGG/ & 288 & $8561879-8561855$ & CM3.5_scaffold00005 & IX $(68-69 \mathrm{cM})$ \\
\hline & CTCCCCAGTGTATCAGCCAAATCTC & & $8561591-8561616$ & & \\
\hline \multirow[t]{2}{*}{ CMMS33-2 } & GCTACTTTTTATGGCGGCAGTGACG/ & 265 & $769897-769873$ & CM3.5_scaffold00063 & $\mathrm{X}(30-35 \mathrm{cM})$ \\
\hline & ATTCGGATGATTATTCTTCGCAGTT & & & & \\
\hline \multirow[t]{2}{*}{ CMMS34-10 } & GGGGTGTGAAGCTGAAGGCAAAGTC/ & 350 & $1924471-1924446$ & CM3.5_scaffold00016 & $\mathrm{X}(22-24 \mathrm{cM})$ \\
\hline & AAAGGAAGAAGAAGAAAAAGGAGAA & & $1924121-1924145$ & & \\
\hline \multirow[t]{2}{*}{ CMMS33-1 } & TGTAATAGGATGACCAAGGGGAGTT/ & 384 & $7366489-7366461$ & CM3.5_scaffold00004 & XII $(10-13 \mathrm{cM})$ \\
\hline & TTCAGGAGCTACAACAAGATTTCAA & & $7366105-7366129$ & & \\
\hline \multirow[t]{2}{*}{ CMMS35-4 } & ACGGATACATCGAGGAGACTTCATG/ & 110 & $7114119-7114143$ & CM3.5_scaffold00001 & XII (18 cM) \\
\hline & GTCAGCTTCAACCCTTTACTTTTTC & & $7114229-7114204$ & & \\
\hline
\end{tabular}


Supplemental Table 2. Estimated size of simple sequence repeat (SSR) amplified alleles in Brazilian melon accessions and references genotypes.

\begin{tabular}{|c|c|c|c|c|c|c|c|c|c|c|c|c|c|}
\hline \multirow[b]{2}{*}{ Accession } & \multicolumn{13}{|c|}{ SSR marker } \\
\hline & 1 & 2 & 3 & 4 & 5 & 6 & 7 & 8 & 9 & 10 & 11 & 12 & 13 \\
\hline IA-1 & $0 / 440$ & 186 & $2 / 162$ & $8 / 116$ & $2 / 184$ & $26 / 126$ & $277 / 277$ & $4 / 484$ & $53 / 267$ & $6 / 486$ & $46 / 346$ & $2 / 108$ & $36 / 3$ \\
\hline A-2 & $0 / 440$ & $6 / 186$ & $0 / 170$ & & $2 / 184$ & $4 / 138$ & $277 / 277$ & $4 / 484$ & $3 / 267$ & & $6 / 346$ & & $6 / 336$ \\
\hline A-5 & $0 / 440$ & $6 / 186$ & $6 / 166$ & $14 / 114$ & $2 / 184$ & $8 / 138$ & $277 / 277$ & $84 / 484$ & $53 / 267$ & $8 / 468$ & $6 / 346$ & $04 / 104$ & $12 / 31$ \\
\hline A- 6 & $0 / 440$ & 194 & $/ 170$ & 08/108 & & 26 & $275 / 275$ & $6 / 484$ & $3 / 267$ & & 346 & 102 & $6 / 33$ \\
\hline A-4 & $0 / 440$ & & $6 / 174$ & $14 / 114$ & & $26 / 126$ & $277 / 277$ & & $3 / 263$ & & $6 / 346$ & $100 / 102$ & $? / ?$ \\
\hline-2 & /440 & & 170 & & 84 & & $277 / 277$ & 84 & $/ 263$ & & & & $44 / 344$ \\
\hline-3 & $0 / 440$ & 194 & 5/166 & $14 / 114$ & /184 & /126 & $277 / 277$ & $46 / 484$ & $/ 267$ & & 346 & /102 & $4 / 344$ \\
\hline E-1 & $0 / 440$ & & & & & & $277 / 277$ & $84 / 484$ & & & & & 312 \\
\hline E-2 & $0 / 440$ & & 176 & & $72 / 184$ & & $277 / 277$ & $484 / 484$ & $3 / 267$ & & 346 & 102 & $2 / 312$ \\
\hline E-3 & & & & & & & $277 / 277$ & & & & & & \\
\hline E-4 & 440 & 194 & $/ 170$ & 16 & 84 & 126 & $275 / 275$ & 84 & $/ 263$ & & $/ 350$ & 02 & $2 / 350$ \\
\hline N-5 & & & & & & & $277 / 277$ & & & & 50 & & $6 / 336$ \\
\hline N-6 & & & & & & & $277 / 277$ & & & & & & \\
\hline N-7 & & & & & & & $277 / 277$ & & 67 & & 50 & & $6 / 336$ \\
\hline N-8 & & & & & & & $/ 277$ & & & & & & \\
\hline N-9 & 440 & 86 & $? / ?$ & & 84 & & $277 / 277$ & $484 / 484$ & $? / ?$ & & $5 / 346$ & & $1 / 344$ \\
\hline $3-1$ & & & & & & & $/ 277$ & & & & & & \\
\hline $3-2$ & 446 & & 62 & & & & $277 / 277$ & & 67 & & & & 44 \\
\hline $3-3$ & & & & & & & 277 & & & & & & 336 \\
\hline $3-4$ & /440 & & & & & & $277 / 277$ & & & & & & \\
\hline$E-1$ & & & & & & & $277 / 277$ & & & & & & 336 \\
\hline $\mathrm{E}-2$ & /440 & & & & & & $277 / 277$ & & & & & & \\
\hline A-1 & & & & & & & $281 / 281$ & & & & & & \\
\hline A-2 & 40 & & & & & & $277 / 277$ & & 67 & & & & 344 \\
\hline A-3 & & & & & & & $/ 277$ & & & & & & 44 \\
\hline A-4 & & & & & & & $277 / 277$ & 64 & 67 & & & & 336 \\
\hline A-5 & & & & & & & $277 / 277$ & & & & & & 336 \\
\hline $1-01$ & & & & & & & $? / ?$ & & & & & & \\
\hline $1-02$ & & & & & & & $271 / 279$ & & & & & & 336 \\
\hline $1-03$ & & & & & & & $279 / 279$ & & & & & & \\
\hline 1-PS & & & & & & & $271 / 279$ & & & & & & $12 / 336$ \\
\hline -HD & & & & & & & $279 / 279$ & & & & & & \\
\hline l-KIR & /402 & 86 & 62 & 08 & 76 & 24 & $? / ?$ & 98 & 69 & 68 & 46 & & $12 / 336$ \\
\hline 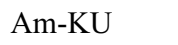 & & & & & 76 & & $? / ?$ & & & & & & $2 / 336$ \\
\hline et-D & & & $162 / 162$ & $114 / 114$ & $? / ?$ & $126 / 126$ & $77 / 277$ & & $263 / 269$ & & & & $12 / 336$ \\
\hline an & $/ 430$ & 86 & 66 & $/ 108$ & $? / ?$ & & $281 / 281$ & 98 & 269 & 68 & 46 & 08 & $312 / 344$ \\
\hline N & & $166 / 166$ & $1 \%$ & & & & $281 / 281$ & & 269 & & & & $312 / 350$ \\
\hline ud-C & $/ 440$ & 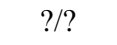 & $66 / 166$ & $14 / 114$ & $184 / 184$ & $126 / 126$ & $277 / 277$ & 98 & $263 / 269$ & $/ 460$ & 54 & 08 & $312 / 336$ \\
\hline$\sqrt{10101}$ & & & & $108 / 114$ & & & $277 / 277$ & & & & & & $312 / 344$ \\
\hline Mom- & $0 / 430$ & $186 / 186$ & $162 / 162$ & $114 / 114$ & $176 / 176$ & $126 / 126$ & $277 / 277$ & $436 / 498$ & $267 / 273$ & 460 & 58 & $100 / 106$ & $312 / 344$ \\
\hline & & 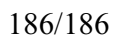 & & & & & $277 / 277$ & & & & & & \\
\hline 4ak-GM & $440 / 440$ & ?/? & $162 / 162$ & $108 / 108$ & $176 / 176$ & $126 / 126$ & $279 / 279$ & $446 / 498$ & $263 / 269$ & & $350 / 350$ & $106 / 112$ & $312 / 350$ \\
\hline 1. & $440 / 440$ & $30 / 180$ & $162 / 162$ & $108 / 108$ & $172 / 172$ & $126 / 126$ & $279 / 279$ & $436 / 498$ & $263 / 269$ & $486 / 486$ & $350 / 350$ & $106 / 112$ & $312 / 350$ \\
\hline & & & & & & & & & & & & & \\
\hline
\end{tabular}


Supplemental Table 2. Continued.

\begin{tabular}{|c|c|c|c|c|c|c|c|c|c|c|c|c|c|}
\hline \multirow[b]{2}{*}{ Accession } & \multicolumn{13}{|c|}{ SSR marker ${ }^{\mathrm{z}}$} \\
\hline & 1 & 2 & 3 & 4 & 5 & 6 & 7 & 8 & 9 & 10 & 11 & 12 & 13 \\
\hline Tib-TIB & $430 / 430$ & $194 / 194$ & $162 / 162$ & $114 / 114$ & $? / ?$ & $136 / 136$ & $275 / 275$ & $384 / 484$ & $267 / 273$ & $486 / 486$ & $346 / 346$ & $102 / 108$ & $350 / 364$ \\
\hline Chi-VELL & $402 / 402$ & $166 / 166$ & $? / ?$ & $? / ?$ & $184 / 184$ & $136 / 136$ & $271 / 271$ & $436 / 484$ & $267 / 269$ & $468 / 468$ & $346 / 346$ & $102 / 108$ & $336 / 350$ \\
\hline Ag-WCh & $402 / 402$ & $180 / 180$ & $162 / 162$ & $108 / 108$ & $172 / 172$ & $124 / 124$ & $279 / 279$ & $436 / 498$ & $263 / 269$ & $468 / 468$ & $350 / 358$ & $102 / 108$ & $336 / 344$ \\
\hline
\end{tabular}

${ }^{\mathrm{z}} 1$ = CMMS 2-3, 2 = CMMS 4-3, 3 = CMMS 12-6, 4 = CMMS 15-4, 5 = CMMS 22-2, 6 = CMMS 24-3, 7 = CMMS 30-3, $8=$ CMMS 33-1, $9=$ CMMS 33-2, 10 = CMMS 34-8, 11 = CMMS 34-10, 12 = CMMS 35-4, 13 = CMMS 35-5. 